\title{
Source rock geochemistry, petrography of reservoir horizons and origin of natural gas in the Devonian of the Lublin and Lviv basins (SE Poland and western Ukraine)
}

\author{
Natalia Y. RADKOVETS ${ }^{1, *}$, Maciej J. KOTARBA² and Krystian WÓJCIK ${ }^{3}$ \\ 1 National Academy of Sciences of Ukraine, Institute of Geology and Geochemistry of Combustible Minerals, Naukova 3a, \\ 79060 Lviv, Ukraine \\ 2 AGH University of Science and Technology, Faculty of Geology, Geophysics and Environmental Protection, \\ al. Mickiewicza 30, 30-059 Kraków, Poland \\ 3 Polish Geological Institute - National Research Institute, Rakowiecka 4, 00-975 Warsaw, Poland
}

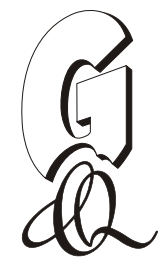

\begin{abstract}
Radkovets, N.Y., Kotarba, M., Wójcik, K., 2017. Source rock geochemistry, petrography of reservoir horizons and origin of natural gas in the Devonian of the Lublin and Lviv basins (SE Poland and western Ukraine). Geological Quarterly, 61 (3): 569-589, doi: 10.7306/gq.1361

The Rock-Eval source rock characteristics, mineral composition and type-porosity of reservoir horizons, and origin of natural gas in the Devonian of the Lublin and Lviv basins are described. In the Lower Devonian, the TOC content ranges from 0.01 to $1.82 \mathrm{wt.} \%$ in the Lublin Basin, and from 0.01 to $0.45 \mathrm{wt} . \%$ in the Lviv Basin. Transformation of organic matter varies from immature in the Lochkovian (Lviv Basin) to mature and overmature in the Emsian (Lublin Basin). The organic matter contains mainly Type-II kerogen, and underwent primary and/or secondary oxidation processes. In the Middle Devonian, the TOC content varies from 0.00 to $1.63 \mathrm{wt} . \%$ in the Lublin Basin, and from 0.02 to 0.64 to $2.35 \mathrm{wt} . \%$ in the Lviv Basin. The organic matter contains mainly Type-II kerogen and is immature in the Givetian of the Lviv Basin and mature in the Eifelian of the Lviv Basin and in the Eifelian and Givetian in the Lublin Basin. In the Upper Devonian, the TOC content is from 0.02 to $2.62 \mathrm{wt} . \%$ in the Lublin Basin, and from 0.04 to $1.43 \mathrm{wt} . \%$ in the Lviv Basin. Type-Il kerogen dominates in both basins. Organic matter is mature in the Upper Devonian in the Lublin Basin and in the Famennian of the Lviv Basin and overmature in the Frasnian of the Lviv Basin. The reservoir horizons in the Devonian of the Lublin and Lviv basins are developed in clastic, carbonate and sulphate rocks. Terrigenous rocks form several separate horizons in the Lower and Middle Devonian of the Lviv Basin, and in the Upper Devonian (Famennian) of the Lublin Basin. Their filtration properties relate to intergranular porosity, while the fracture space has subordinate significance. Carbonate rocks form thick saturated horizons in the Givetian in the Lviv Basin, and in the Eifelian, Givetian and Frasnian in the Lublin Basin. Their filtration properties are produced by fracture porosity. Sulphates and carbonate-sulphate rocks with fracture and cavern porosity play a role as reservoir horizons in the Middle Devonian of the Lublin Basin. The natural gas collected from the Upper Devonian of the Lublin Basin was generated mainly during low-temperature thermogenic processes, from Ordovician-Silurian Type-II kerogen. The gas from the Middle Devonian reservoirs of the Lviv Basin was produced from Ordovician-Silurian Type-II kerogen and partly from the Middle and Upper Devonian mixed Type-III/II kerogen with maturity from about 0.9 to $1.4 \%$. Carbon dioxide was formed by both thermogenic and microbial processes. Molecular nitrogen was generated mainly through thermal transformation of organic matter and also from destruction of $\mathrm{NH}_{4}$-rich illite of the clayey facies of the Ordovician-Silurian strata.
\end{abstract}

Key words: Devonian, Lublin Basin, Lviv Basin, Rock-Eval pyrolysis, petrography, isotope geochemistry.

\section{INTRODUCTION}

Since the 1960s several gas and oil fields have been discovered, and numerous gas shows observed, in the Devonian strata of the Lublin and Lviv basins (Karnkowski, 1999; Helcel-Weil and Dzięgielowski, 2003). In Ukraine, a number of oil and gas shows have been tested in the Lokachi, Olesko, Gorokhiv and Oglyadiv boreholes, and two gas fields (Lokachi

*Corresponding author, e-mail: radkov_n@ukr.net

Received: October 27, 2016; accepted: April 26, 2017; first published online: May 25, 2017 and Velyki Mosty) have been discovered (Khizhniakov, 1975; Krupskiy, 2001; Krupskiy et al., 2014). In Poland, effective gas and oil accumulations have been found in Devonian rocks in the Ciecierzyn, Mełgiew, Komarów and Glinnik boreholes in the Lublin Basin (Fig. 1).

The source rock potential, petrographic properties of reservoir rocks and the origin of natural gas in the Devonian of the western margin of the East European Platform are the subject of this paper. We compare the Rock-Eval characteristics of the source rocks, mineral composition and porosity-type of reservoir horizons, as well as the composition of natural gas in the Devonian between the Lviv and Lublin basins. The origin of hydrocarbon components, carbon dioxide and molecular nitrogen in the natural gas are founded on their molecular and stable isotopic compositions $\left({ }^{12,13} \mathrm{C}\right.$ in $\mathrm{CH}_{4}, \mathrm{C}_{2} \mathrm{H}_{6}, \mathrm{C}_{3} \mathrm{H}_{8}, n \mathrm{C}_{4} \mathrm{H}_{10}, i \mathrm{C}_{4} \mathrm{H}_{10}$ 


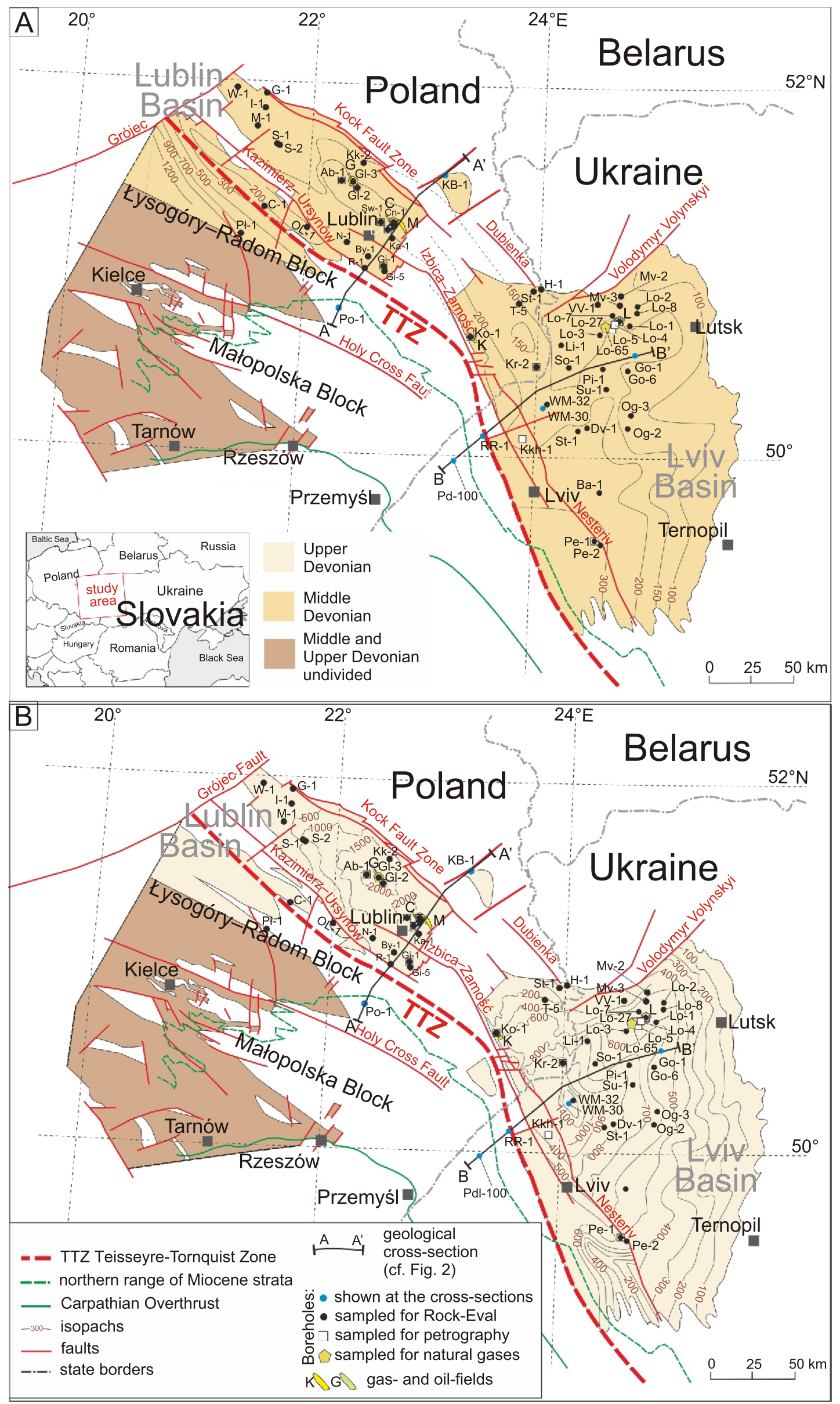


and $\mathrm{CO}_{2},{ }^{1,2} \mathrm{H}$ in $\mathrm{CH}_{4}$, and ${ }^{14,15} \mathrm{~N}$ in $\mathrm{N}_{2}$ ) in the Glinnik oil and gas and Ciecierzyn gas fields in the Lublin Basin, and in the Lokachi gas field in the Lviv Basin.

\section{GEOLOGICAL SETTING AND PETROLEUM OCCURRENCES}

\author{
GEOLOGICAL CHARACTERISTICS OF THE LUBLIN \\ AND LVIV BASINS
}

The Lublin and Lviv basins are SE-trending structural depressions, situated at the SW margin of the East European Craton. The basins are bordered to the SW by the Łysogóry-Radom and Małopolska blocks and the Rava Rus'ka Zone of the Trans-European Suture Zone (TESZ). The Lublin and Lviv basins consist of deformed Riphean-Pennsylvanian strata (e.g., Żelichowski, 1972; Pomyanovskaya, 1974 Miłaczewski, 1981; Żelichowski and Kozłowski, 1983; Kruglov and Tsypko, 1988; Chebanenko et al., 1990; Vashchenko et al., 2007; Narkiewicz, 2007, 2011; Narkiewicz et al., 2011, 2015 Krzywiec et al., 2017; Fig. 2). These attain 10 km in thickness in the Lublin Basin and thin out toward the centre of the East European Craton (Figs. 1 and 2). This succession is overlain by slightly deformed (epi-Variscan) Permian-Mesozoic strata of the German-Polish Basin (see Krzywiec et al., 2017 for references). The NE limit of the basins is defined by the erosional pinch-out of Pennsylvanian strata in the Lublin Basin and of Devonian strata in the Lviv Basin at the sub-Mesozoic subcrop (e.g., Pożaryski and Dembowski, 1983). The SW boundary consists of two regional fault zones: the Ursynów-Kazimierz Fault Zone to the north-west and the Izbica-Zamość-Nesteriv Fault Zone to the south-east. The Teisseyre-Tornquist Zone is slightly to the SW of these (Krzywiec et al., 2017; Fig. 1).

The thickness of the Lower Devonian strata reaches $>2000 \mathrm{~m}$ in the Lublin Basin and up to $1900 \mathrm{~m}$ in the Lviv Basin (Miłaczewski, 1981; Chebanenko et al., 1990; Narkiewicz, 2011, Radkovets, 2016). The succession is composed of limestones, marls, claystones and fine-grained siliciclastic rocks in the lower part, and of continental clastic rocks in the upper part. The Lower Devonian is divided into the Sycyna, Czarnolas and Zwolen formations in the Lublin Basin, which correspond to the Tyver and Dnister series in the Lviv Basin (Fig. 3). Gas accumulations in the Lower Devonian deposits were discovered at Lokachi in the NW part of the Lviv Basin (Fig. 4C).

The Middle Devonian reaches a maximal thickness of $\sim 200 \mathrm{~m}$ in the central part of the Lublin Basin and $>300 \mathrm{~m}$ at the SW margin of the Lviv Basin (Fig. 1A). The succession is composed of carbonates intercalated with clastics and evaporites In the Lublin Basin, the Middle Devonian deposits are included to the Telatyn Formation, which is divided into the Przewodów and Giełczew members in the NW and central part of the basin, and into the Przewodów, Machnów, Żniatyń, Pełcza, Rachanie and Mircza members in the SE part (Fig. 3). In the Lviv Basin, the Middle Devonian succession is divided into the Lopushany, Pelcha and Strutyn suites. The Przewodów, Machnów and Żniatyń members correspond to the Lopushany Suite. The Pełcza Member and Pelcha Suite are lateral equivalents, while the Rachanie and Mircza members correlate with the Strutyn Suite (Fig. 3). Gas accumulations within the Middle Devonian deposits were discovered in Komarów in the SE part of the Lublin Basin and in Lokachi in the NW part of the Lviv Basin (Fig. 4C).

The Upper Devonian strata reach a maximal thickness $>2000 \mathrm{~m}$ in the central part of the Lublin Basin and $>1200 \mathrm{~m}$ in the central part of the Lviv Basin (Fig. 1B).The Frasnian is composed of shallow-marine platform type carbonates. In the Lublin Basin these deposits are included to the Modryn Formation, which is divided into the Krzewica, Lipowiec, Łosien and Zubowice members in the SE part, and into the Łosień, Ciecierzyn, Metgiew and Zubowice members in the central part of the basin (cf. Miłaczewski, 1981; Narkiewicz, 2011). In the Lviv Basin, the Frasnian is divided (from the base to the top of the section) into the Remeziv and Zolochiv suites, and Voronezh, Yevlanovo and Livny horizons. The Krzewica and Lipowiec Members correspond to the Remeziv Suite. The Ciecierzyn and Łosien members correlate with the Zolochiv Suite, while the Zubowice Member corresponds to the Voronezh, Yevlanovo and Livny horizons (Fig. 3). Gas accumulations within the Frasnian deposits were discovered in Ciecierzyn field (Zubowice Member) and Mełgiew field (Melgiew Member) in the central part of the Lublin Basin (Fig. 4B).

The Famennian is composed of basinal fine-grained clastic-carbonate rocks in the lower part, and of lagoonal-continental clastic rocks in the upper part. In the central and NE part of the Lublin Basin, the Famennian succession is divided into the Bychawa and Firlej formations, Niedrzwica Beds, Hulcze Formation and a succession of variegated clastic rocks (Narkiewicz, 2011). The Firlej and Hulcze formations occur in the SE part of the basin. They correspond to the Zadon, Yelets, Litovezh, Zakhidnyi Bug and Volodymyr Volynskyi units in the Lviv Basin (Fig. 3). Gas accumulations in the Famennian Bychawa and Hulcze formations were discovered in the Glinnik (Fig. 4A) and Ciecierzyn (Fig. 4B) fields, in the central part of the Lublin Basin.

\section{PETROLEUM OCCURRENCES}

So far, 4 gas-fields and one oil-field have been discovered in the Devonian of the Lublin Basin. These are as follows: Ciecierzyn, Mełgiew A, Mełgiew B, Komarów and Glinnik fields. Another 2 gas accumulations - of Lokachi and Velyky Mosty occur in the Devonian of the Lviv Basin.

Fig. 1. Sub-Carboniferous map of the Middle (A) and Upper (B) Devonian subcrops in the southwestern margin of the East European Craton (modified after Medvedyev, 1979; Pożaryski and Dembowski, 1983; Żelichowski and Kozłowski, 1983; Chebanenko et al., 1990; Miłaczewski, 2010; Narkiewicz et al., 2015; Krzywiec et al., 2017) with location of Devonian oil- and gas-fields and boreholes studied

Boreholes: Lviv Basin: Ba - Baluchyn, Dv - Dobrotvir, Go - Gorokhiv, Kkh - Krekhiv, Li - Litovezh, Lo - Lokachi, Mv - Mynkiv, Og Oglyadiv, Pdl - Pidluby, Pe - Peremyshlyany, Pi - Pidberezzia, RR - Rava Ruska, So - Sokal, Su - Sushne, St - Stremin, VV Volodymyr-Volynsky, WM - Velyki Mosty; Lublin Basin: Ab - Abramów, By - Bychawa, C - Ciepielów, Cn - Ciecierzyn, G - Garwolin, Gi Giełczew, GI - Glinnik, H - Horodło, I - Izdebno, Ka - Kawęczyn, KB - Krowie Bagno IG, Kk - Kock, Ko - Komarów, Kr-Korczmin, L - Lublin, M - Maciejowice, N - Niedrzwica, OL - Opole Lubelskie, Pł - Płusy, Po - Potok IG, R - Rudnik, S - Stężyca, St - Strzelce, Sw - Świdnik, T Terebin, W - Wilga (between the Lublin and Ciecierzyn boreholes there are also Krępiec-1, Minkowice-4a, and Mełgiew-2). Oil- and gas-fields: C - Ciecierzyn, G - Glinnik, M - Mełgiew A and Mełgiew B undivided, K - Komarów, L - Lokachi 


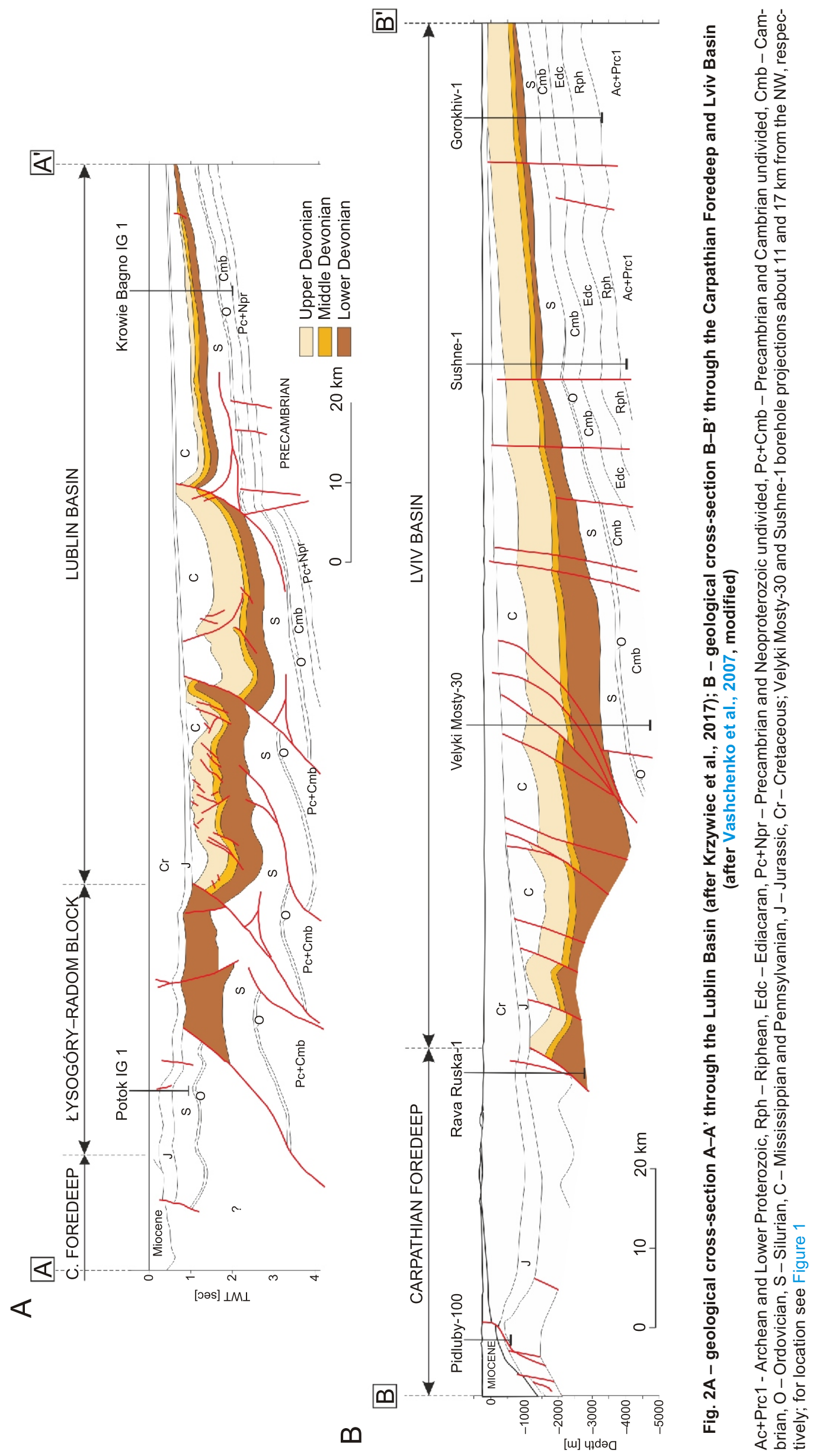

田 
The Glinnik oil and gas field is located in the central part of the Lublin Basin (Fig. 1). The oil accumulation was discovered in 1990 in the Upper Devonian (Famennian) sandstones of the Hulcze Formation (Fig. 4A). The reservoir rocks occur at depths between 3293.0 and $3300.0 \mathrm{~m}$, ranging from 1.5 to $7.0 \mathrm{~m}$ in thickness. The Glinnik anticlinal-type trap was penetrated by 3 boreholes: Glinnik-1, -3 and -4 . The oil-field surface is $0.6 \mathrm{~km}^{2}$ and current resources are estimated at $5,000 \mathrm{t}$ of oil and $0.0006 \mathrm{bn} \mathrm{m}^{3}$ of gas. Hydrocarbon accumulations are sealed by Mississippian (Visean) claystones and siltstones.

The Ciecierzyn gas field is located in the central part of the Lublin Basin, $\sim 20 \mathrm{~km}$ SE from the Glinnik field (Fig. 1). The effective gas accumulation was discovered in 1984 in the Upper Devonian (Frasnian) dolomites of the Werbkowice Member (sensu Miłaczewski, 1981) in the Modryń Formation (Fig. 4B). The main gas horizon occurs at depths between 3736.0 and $3820.0 \mathrm{~m}$ and ranges from 33 to $80 \mathrm{~m}$ in thickness. A smaller gas-saturated horizon in the Ciecierzyn field occurs in the Famennian marly limestones of the Bychawa Formation at depths between 2449.0 and $3431.0 \mathrm{~m}$. Small hydrocarbon shows appear also within the Famennian sandstones of the Hulcze Formation at depths between 1560.0 and $1590.0 \mathrm{~m}$. Neither of these Famennian horizons have been exploited. The Ciecierzyn anticlinal-type trap was penetrated by 3 boreholes: Ciecierzyn-1, -2 and -3 . The gas-field surface is $6.5 \mathrm{~km}^{2}$ and current gas resources are estimated at $0.450 \mathrm{bn} \mathrm{m}^{3}$. The main gas-bearing horizon in the Ciecierzyn field is sealed by carbonates of the Zubowice Member (Fig. 4B). The two younger horizons are sealed by the Famennian marls and Mississippian (Visean) claystones and siltstones, respectively.

Apart from the two fields mentioned above, the Mełgiew and Komarów gas-fields also occur in the Lublin Basin. The Mełgiew field (divided into the Mełgiew A and B fields) is located in the central part of the Lublin Basin and was penetrated by 9 boreholes. The gas accumulations were discovered in 1991 in the Upper Devonian (Frasnian) dolomites (Mełgiew A) and limestones (Mełgiew B) of the Modryń Formation (Helcel-Weil and Dziegielowski, 2003). The Komarów gas field was located in the southeastern part of the Lublin Basin and was penetrated by 9 boreholes. The effective gas accumulation was discovered in 1967 in the Middle Devonian (Givetian) limestones, dolomites anhydrites and sandstones of the Telatyń Formation. Its exploitation ended in 1979.

The Lokachi gas-field is located in the northeastern part of the Lviv Basin. The effective gas accumulations were discovered in 1979 in one horizon of the Lower Devonian sandstones of the Tyver Series (Lochkovian) and in 6 horizons in the Middle Devonian (Eifelian, Givetian) - sandstones, biodetrital dolomitised limestones and dolomitites of the Lopushany, Pelcha and Strutyn suites (Fig. 4C). The reservoir rocks occur at depths between 815.0 and $1200.0 \mathrm{~m}$, ranging from 3.2 to $7.8 \mathrm{~m}$ in thickness (Fedyshyn, 1998). The gas column is $182 \mathrm{~m}$ at the field, ranging in separate horizons from 34 to $94 \mathrm{~m}$. Gas accumulations are sealed by sulphate and carbonate-sulphate rocks, which range in thickness from 15 to $20 \mathrm{~m}$. The Lokachi gas field is penetrated by 28 boreholes, while gas-bearing horizons have been discovered in 21 of them (Lokachi-2, -5, -7,-27, $-51,-53,-54,-55,-56,-57,-58,-59,-61,-62,-63,-64,-65,-66$, $-67,100$ and -101$)$. The gas-field surface is $38.5 \mathrm{~km}^{2}$ and initial resources are estimated at $6972 \mathrm{~m} \mathrm{~m}^{3}$ of gas (Fedyshyn, 1998; Galabuda et al., 2007).
Apart from the Lokachi gas field, there is also the Velyki Mosty gas field, located in the NW part of the Lviv Basin. The Velyki Mosty gas field was penetrated by 31 boreholes, two of which have yielded commercial gas. The field was discovered in 1964 in the Middle Devonian (Givetian) terrigenous rocks of the Lopushany Suite.

\section{METHODOLOGY}

\section{ROCK SAMPLING SITES AND PROCEDURE OF ROCK-EVAL ANALYSES}

In total, 69 rock samples were collected from the Devonian of the Lublin Basin. They were taken from seven boreholes: Abramów-1, Ciecierzyn-1, Giełczew IG 1, Glinnik-2, Komarów IG 1, Korczmin IG 2 and Świdnik IG 1. Another 51 Devonian rock samples have been collected from the Lviv Basin (Ukrainian part of the study area). They were taken from nineteen boreholes: Baluchyn-1, Dobrotvir-1, Gorokhiv-6, Litovyzh-1, Lokachi-2, -3, $-7,-8,-27$, Mlynkiv-3, Ogladiv-2, Peremyshlyany-1, -2, Pidberezzya-1, Sokal-1, Stremin-1, Sushne-1, Velyki Mosty-32, Volodymyr Volynsky-1. The source rock potential is based on Rock-Eval 6 pyrolysis analyses. Samples have been collected only from unweathered parts of the cores, then they were cleaned with a brush to remove mud contamination and pulverized to $<0.2 \mathrm{~mm}$. A list of analysed rock samples and results of Rock-Eval analyses is given in Appendix $1^{*}$.

We used also the previous results of Rock-Eval-II pyrolysis analyses provided by Kotarba et al. $(1998,2005)$. These include 376 Devonian samples derived from twenty six boreholes from the Lublin Basin (Bychawa IG 1, Ciepielów IG 1, Garwolin-1, Giełczew PIG 5, Horodło-1, Izdebno IG 1, Kawęczyn-1, Kock IG 2, Komarów IG 1, Krępiec-1, Krowie Bagno IG 1, Lublin IG 1, Maciejowice IG 1, Mełgiew-2, Minkowice-4a, Niedrzwica IG 1, Opole Lubelskie IG 1, Płusy IG 1, Rudnik IG 1, Stężyca-1, Stężyca-2, Strzelce IG 2, Szwejki IG 3, Świdnik IG 1, Terebin IG 5 and Wilga IG 1).

In total, we used the results of 445 Rock-Eval pyrolysis measurements from the Lublin Basin and 51 measurements from the Lviv Basin for the geochemical characteristics of the Devonian rocks. The location of the boreholes analysed is shown in Figure 1, while the stratigraphic and depth positions of samples are illustrated in Figure 3.

\section{ROCK SAMPLING SITES AND PROCEDURE} OF PETROGRAPHIC ANALYSES

In total, 65 rock samples from 15 boreholes have been analysed (Appendix 2), with 32 samples taken from 8 boreholes in the Lublin Basin (Abramów-1, Ciecierzyn-1, Giełczew IG 1, Glinnik-2, Komarów IG 1, Korczmin IG 2, Lublin IG 1 and Świdnik IG 1), and 33 samples were collected from 5 boreholes in the Lviv Basin (Krekhiv-1, Lokachi-4, -5 and -27 and Peremyshlyany-1; see Figs. 1 and 3 for location).

The $\mathrm{CaCO}_{3}$ and $\mathrm{CaMg}\left(\mathrm{CO}_{3}\right)_{2}$ rock-content were calculated from chemical analyses, performed at the Institute of Geology and Geochemistry of Combustible Minerals. Mineral carbon content was measured using Rock-Eval pyrolysis (Table 1). Thin sections were examined under a Carl Zeiss Jena polarizing microscope.

\footnotetext{
* Supplementary data associated with this article can be found, in the online version, at doi: 10.7306/gq.1361
} 


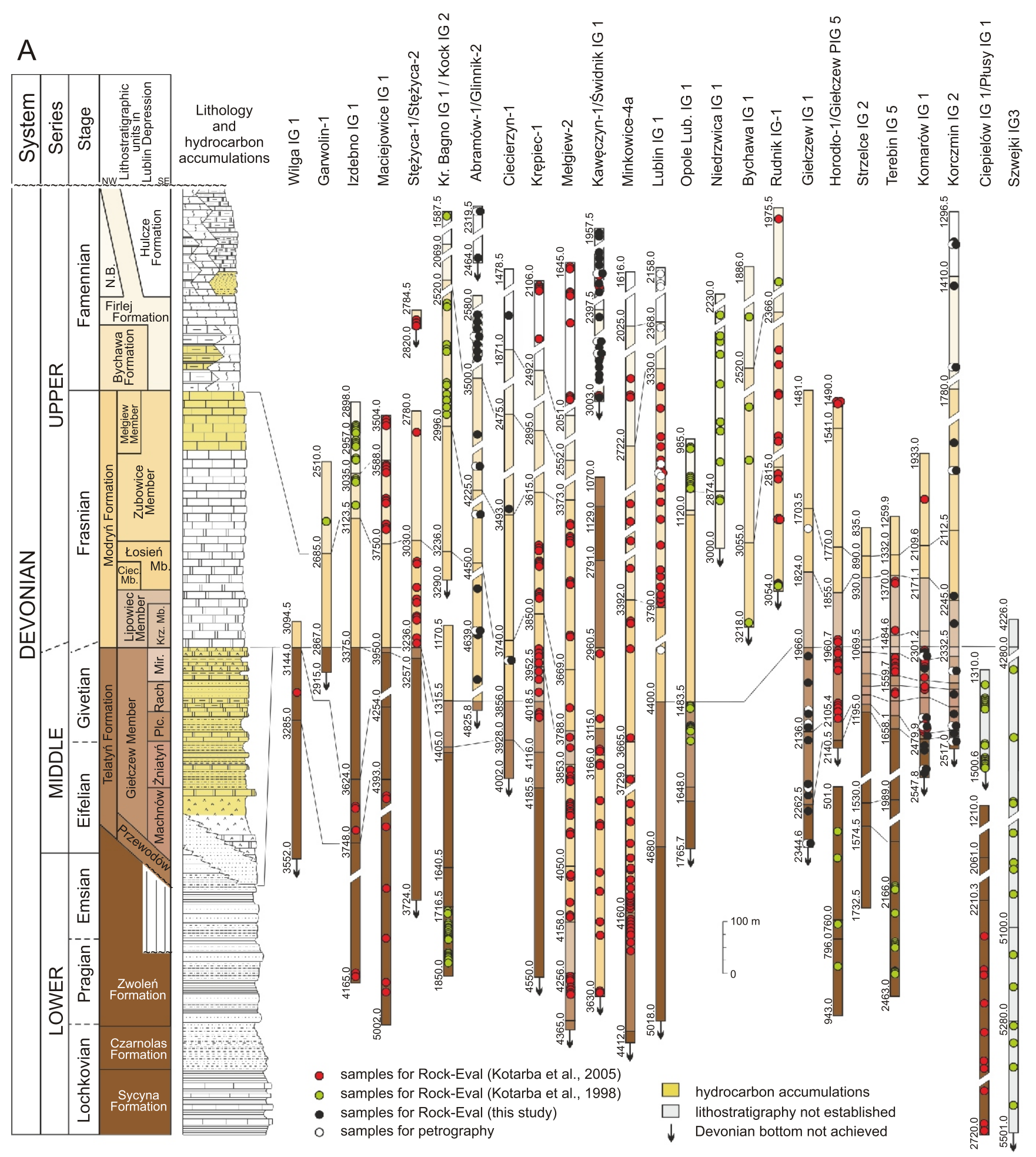

Fig. 3A - stratigraphy, correlation and hydrocarbon potential of the Devonian in the Lublin Basin with location of the samples hydrocarbon accumulations after Helcel-Weil and Dzięgielowski (2003); B - stratigraphy, correlation and hydrocarbon potential stratigraphy and lithology after Pomyanovskaya (1974); 


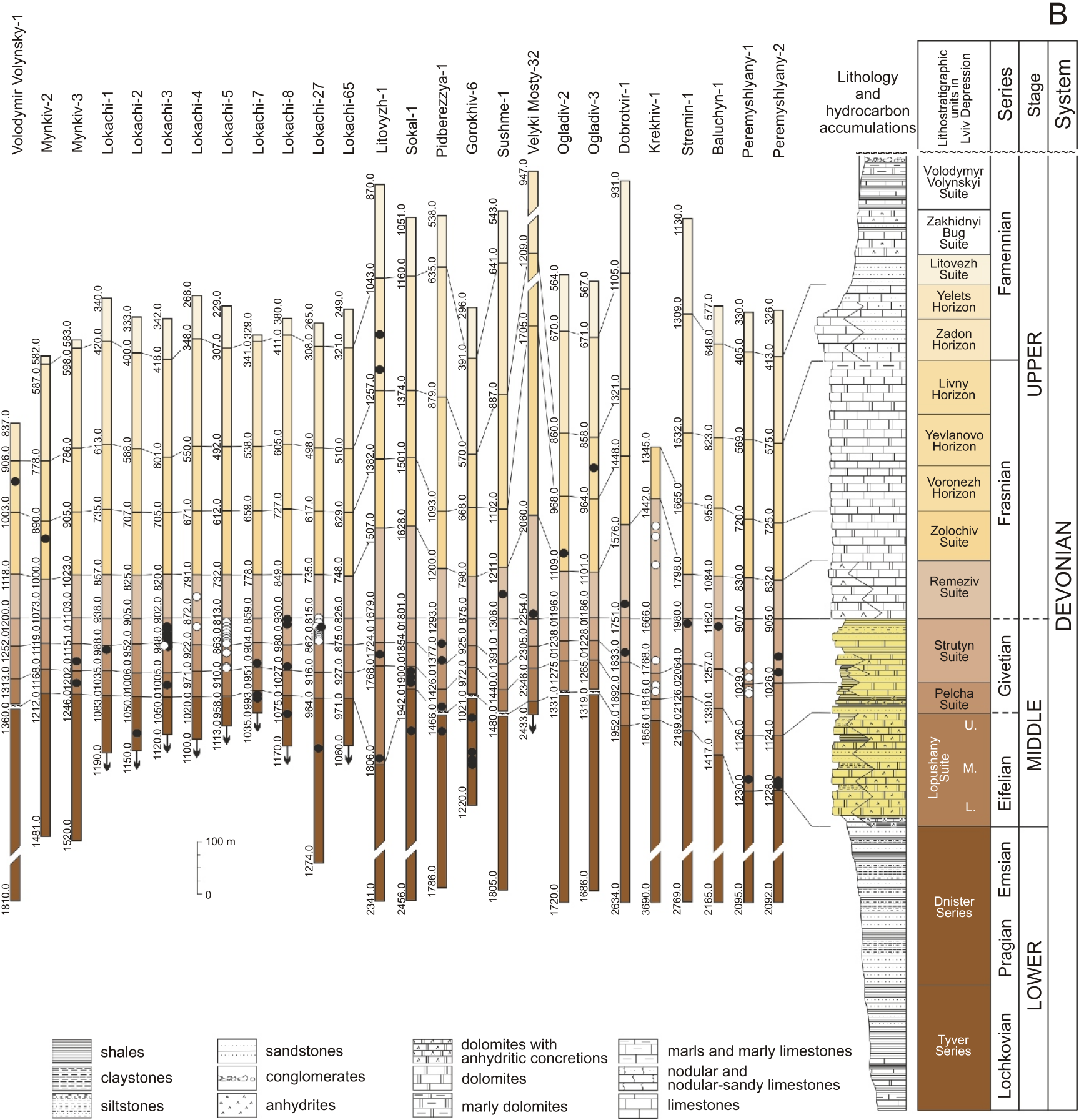

studied in the boreholes analysed; stratigraphy and lithology after Miłaczewski (1981) and Narkiewicz (2011); of the Devonian in the Lviv Basin with location of the samples studied in the boreholes analysed;

hydrocarbon accumulations after Krupskiy et al. (2014)

Mir. - Mircza Member, Rach. - Rachanie Member, Plc. - Pełcza Member 

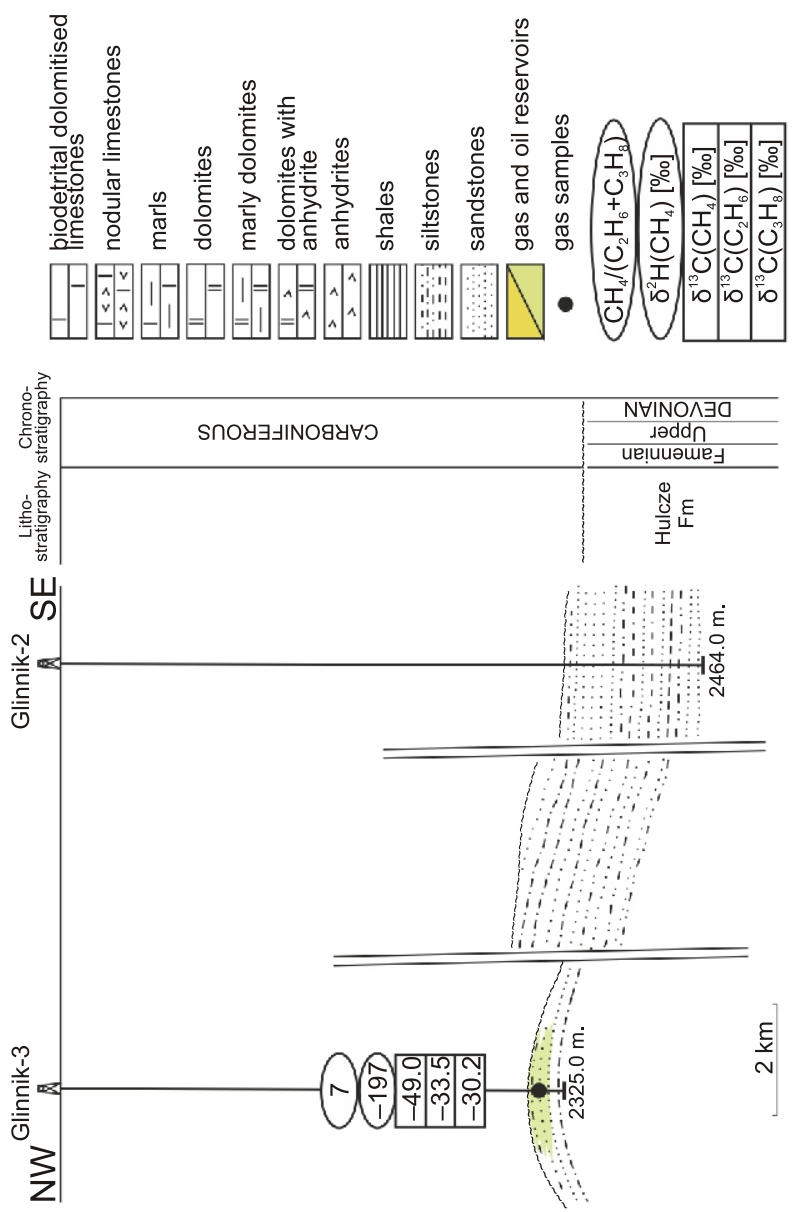

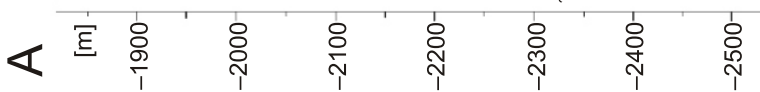
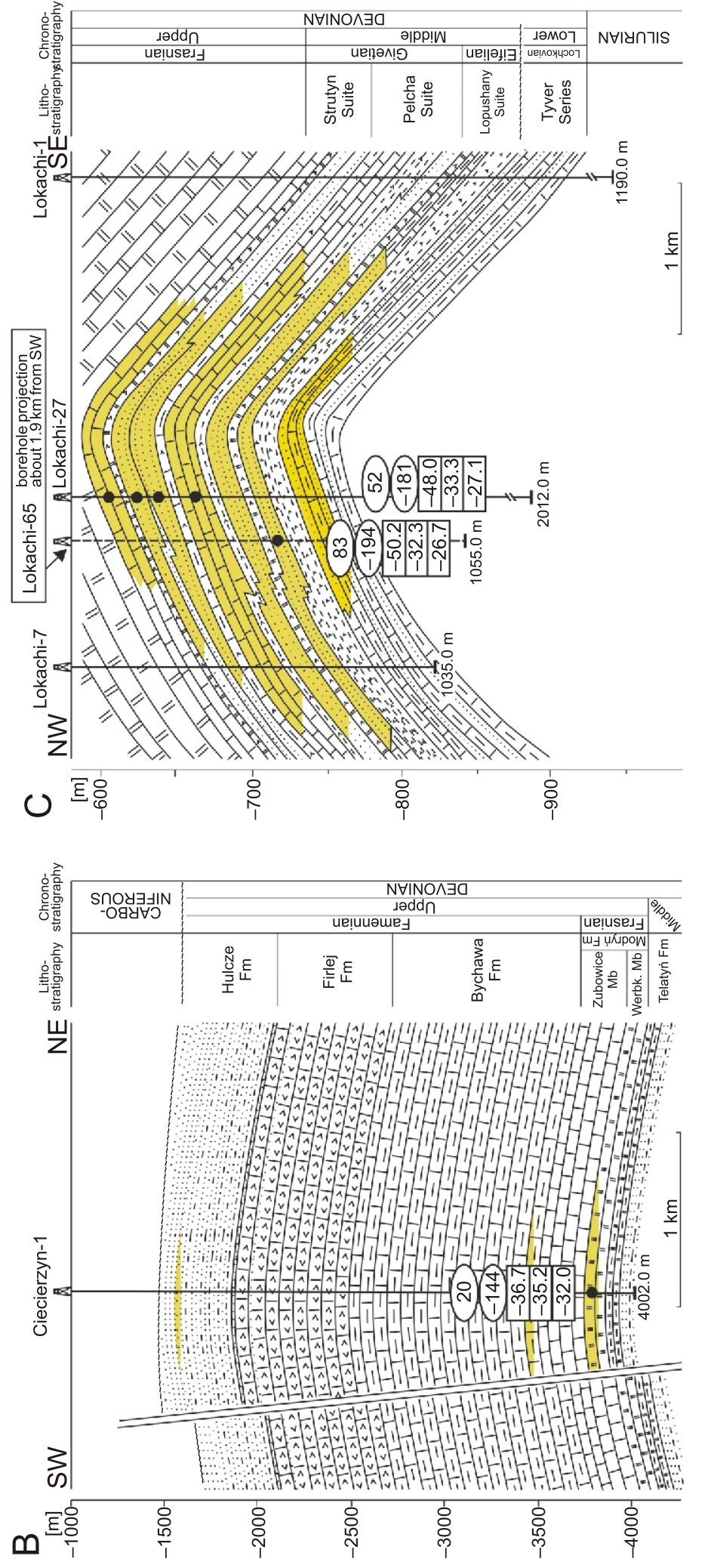

音

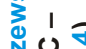

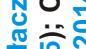

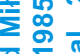
实

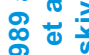

竞这

政

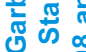

屯 。

恋京

은 $\stackrel{\circ}{\varepsilon}$

宅。

융

훈 훙

웧ำ

\&

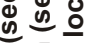

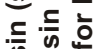

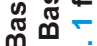

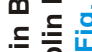

竞高

0

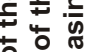

둥

䨔

잉

(

政

.

흥 흥

is

의의

旅

능

원

.

허웡

흑항

列

학

을 은 등

至

등

造

is

政.

정응 응

\%

응 1

ब‥ 


\begin{tabular}{|c|c|c|c|c|c|c|c|c|c|c|c|c|}
\hline \multirow{4}{*}{\multicolumn{2}{|c|}{ 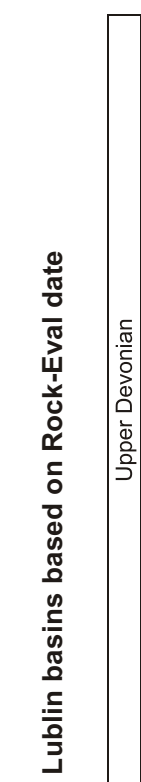 }} & \multirow{2}{*}{ 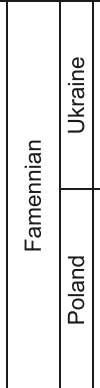 } & $\begin{array}{l}\text { NT- } \\
\frac{N}{0} \mid \\
0 \\
1 \\
\vdots \\
0 \\
0\end{array}$ & 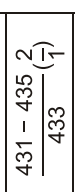 & 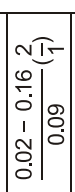 & 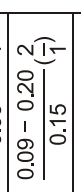 & 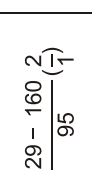 & $\begin{array}{l}\text { NT } \\
\text { N } \\
1 \\
\vdots \\
\end{array} \mid \stackrel{N}{N}$ & 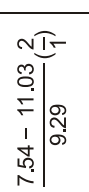 & $\stackrel{0}{\dot{E}}$ & 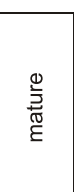 & $\stackrel{\dot{\leftrightarrow}}{\dot{I}}$ \\
\hline & & & 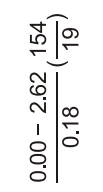 & 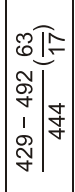 & 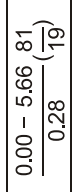 & 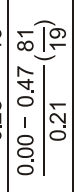 & 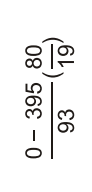 & 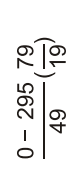 & 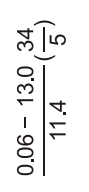 & $\stackrel{\text { 兰 }}{ }$ & 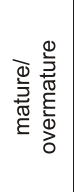 & 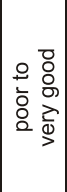 \\
\hline & & $\mid$ & 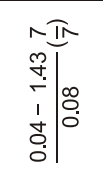 & 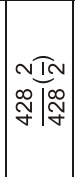 & 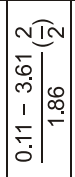 & 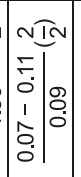 & 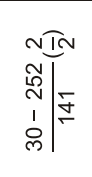 & 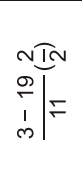 & 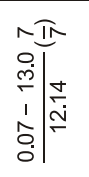 & $\stackrel{\text { 竎 }}{ }$ & 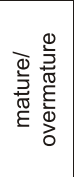 & 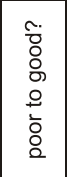 \\
\hline & & $\mid$\begin{tabular}{c|}
$\frac{\mathrm{g}}{\mathrm{c}}$ \\
$\mathrm{w}$ \\
$\mathrm{o}$ \\
$\mathrm{o}$
\end{tabular} & 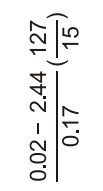 & 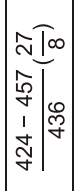 & 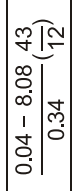 & 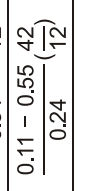 & 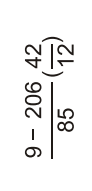 & 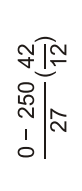 & 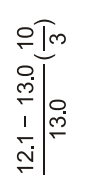 & 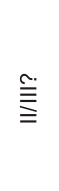 & 总 & 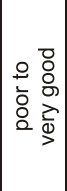 \\
\hline 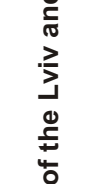 & \multirow[b]{2}{*}{ 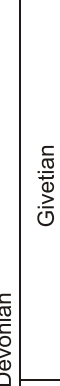 } & 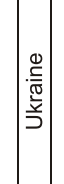 & 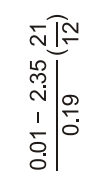 & 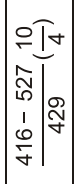 & 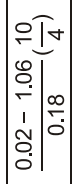 & 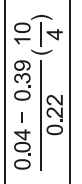 & 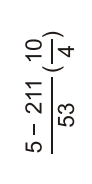 & 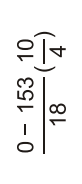 & 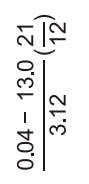 & 竎 & 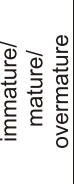 & 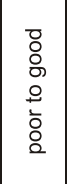 \\
\hline 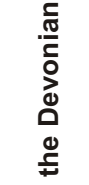 & & $\mid \begin{array}{l}\frac{7}{c} \\
\frac{\mathrm{m}}{0} \\
0\end{array}$ & 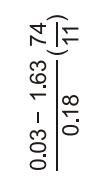 & 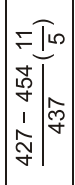 & 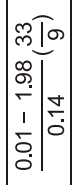 & 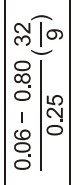 & 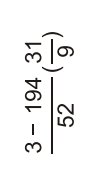 & 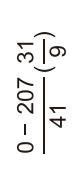 & 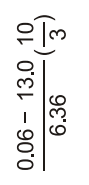 & 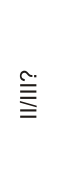 & 总 & 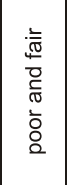 \\
\hline 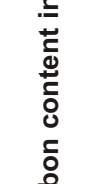 & 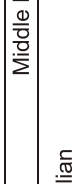 & 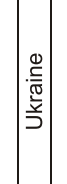 & 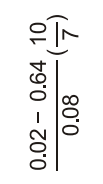 & 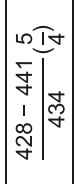 & 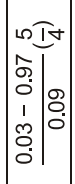 & 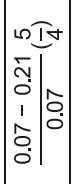 & 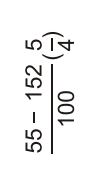 & 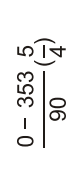 & $\begin{array}{l}0 \pi \\
0 . \pi \\
0 \\
\infty \\
0 \\
1 \\
0 \\
0 \\
0 \\
0\end{array}$ & $\stackrel{\text { 竎 }}{ }$ & 竞 & 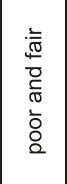 \\
\hline 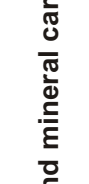 & 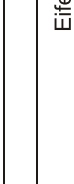 & 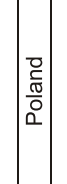 & 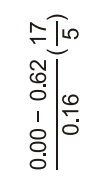 & 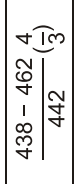 & 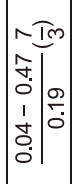 & 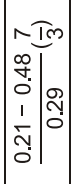 & $\begin{array}{l}\hat{N} \\
\hat{N} \\
1 \\
1 \\
m\end{array}$ & 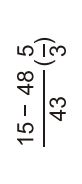 & 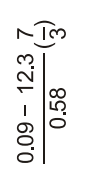 & $\stackrel{\text { 兰 }}{ }$ & 总 & 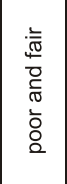 \\
\hline 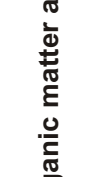 & 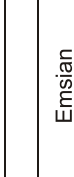 & $\begin{array}{l}\frac{\pi}{\mathrm{c}} \\
\frac{\mathrm{c}}{\mathrm{c}} \\
\mathrm{a}\end{array}$ & 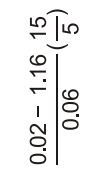 & 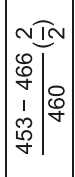 & 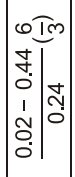 & 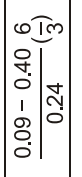 & 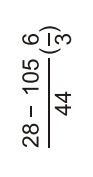 & $\begin{array}{l}n=m \\
\infty \\
\infty \\
1 \\
\vdots\end{array} \mid \mathbb{N}$ & $\left.\begin{array}{l}\infty \text { Im } \\
0 \\
0 \\
0 \\
1 \\
1 \\
0 \\
0 \\
0\end{array}\right|^{\infty}$ & 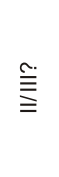 & 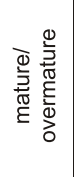 & 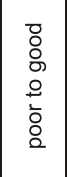 \\
\hline 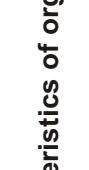 & 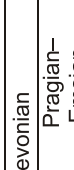 & $\mid$ & 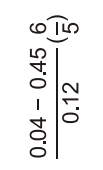 & 1 & 1 & 1 & 1 & 1 & 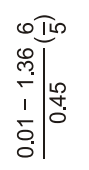 & $\stackrel{0}{\dot{I}}$ & $\stackrel{\dot{S}}{\dot{I}}$ & $\begin{array}{l}\dot{\dot{E}} \\
\dot{J}\end{array}$ \\
\hline 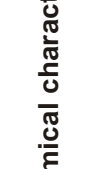 & 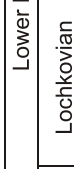 & 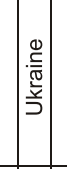 & 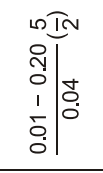 & 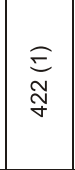 & $\begin{array}{l}\underset{\Xi}{\tilde{O}} \\
\stackrel{0}{0}\end{array}$ & 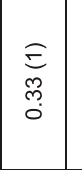 & $\begin{array}{l}\text { E } \\
\stackrel{0}{0}\end{array}$ & $\underset{\substack{\infty \\
m}}{E}$ & $\begin{array}{l}n \text { IN } \\
0 \\
0 \\
m \\
1 \\
b \\
0 \\
0 \\
0\end{array}$ & $\stackrel{\dot{g}}{\dot{E}}$ & 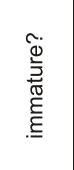 & 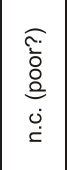 \\
\hline $\begin{array}{l}\bar{c} \\
\dot{0} \\
0 \\
0\end{array}$ & 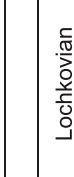 & $\begin{array}{l}\frac{\mathrm{c}}{\mathrm{c}} \\
\mathrm{c} \\
\mathrm{c} \\
\mathrm{a} \\
\end{array}$ & 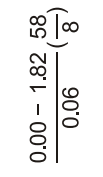 & 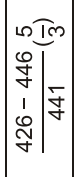 & 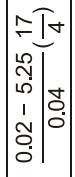 & 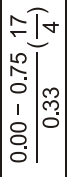 & 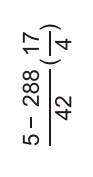 & 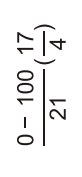 & 1 & $\stackrel{\text { 总 }}{=}$ & 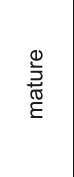 & \begin{tabular}{|l|} 
\\
0 \\
$o$ \\
$o$ \\
$o$ \\
$\vdots$ \\
$\vdots$ \\
0
\end{tabular} \\
\hline & 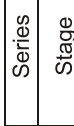 & & & 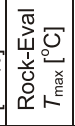 & 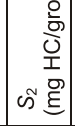 & {$[0 .=1$} & & & & 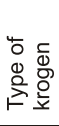 & $\begin{array}{l}\text { 衰 } \\
\text { 焉 }\end{array}$ & 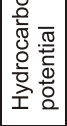 \\
\hline
\end{tabular}


Location of gas boreholes sampled

\begin{tabular}{|c|c|c|c|c|c|c|c|c|c|}
\hline \multirow{2}{*}{ Borehole } & \multirow{2}{*}{$\begin{array}{l}\text { Sam- } \\
\text { ple } \\
\text { code }\end{array}$} & \multirow{2}{*}{ Field name } & \multirow{2}{*}{$\begin{array}{l}\text { Stratigraphy } \\
\text { of reservoir }\end{array}$} & \multirow{2}{*}{$\begin{array}{l}\text { Name of } \\
\text { horizon }\end{array}$} & \multirow{2}{*}{$\begin{array}{l}\text { Surface } \\
{[\mathrm{m} \text { a.s.l.] }}\end{array}$} & \multirow{2}{*}{$\begin{array}{l}\text { Reservoir } \\
\text { depth } \\
\text { [m b.s.l.] }\end{array}$} & \multirow{2}{*}{$\begin{array}{l}\text { Type of gas } \\
\text { accumulation }\end{array}$} & \multicolumn{2}{|c|}{ Coordinates } \\
\hline & & & & & & & & Latitude (N) & Longitude (E) \\
\hline \multicolumn{10}{|c|}{ Lviv Basin } \\
\hline Lokachi-27 & Lo-27 & Lokachi & $\begin{array}{l}\text { M. Devonian } \\
\text { - Givetian }\end{array}$ & P.\&S. & 210 & $815-870$ & free & $50^{\circ} 46^{\prime} 27^{\prime \prime}$ & $24^{\circ} 41^{\prime} 15^{\prime \prime}$ \\
\hline Lokachi-65 & Lo-65 & Lokachi & $\begin{array}{l}\text { M. Devonian } \\
\text { - Eifelian }\end{array}$ & Lop. & $230^{* *}$ & 938-956 & free & $50^{\circ} 43^{\prime} 15^{\prime \prime}$ & $24^{\circ} 43^{\prime} 46^{\prime \prime}$ \\
\hline \multicolumn{10}{|c|}{ Lublin Basin } \\
\hline Ciecierzyn-1 & $\mathrm{Cn}-1$ & Ciecierzyn & $\begin{array}{l}\text { U. Devonian } \\
\text { - Frasnian }\end{array}$ & & 176 & $3740-3820$ & free & $51^{\circ} 16^{\prime} 52^{\prime \prime}$ & $22^{\circ} 36^{\prime} 10^{\prime \prime}$ \\
\hline Glinnik-3 & GI-3 & Glinnik & $\begin{array}{l}\text { U. Devonian } \\
\text { - Frasnian }\end{array}$ & & 156 & $2293-2325$ & dissolved & $51^{\circ} 25^{\prime} 37^{\prime \prime}$ & $22^{\circ} 22^{\prime} 09^{\prime \prime}$ \\
\hline
\end{tabular}

U. - Upper, M. - Middle, Lop. - Lopushany Suite, P.\&S. - Pelcha and Strutyn Suite, a.s.I. - above sea level, b.s.I. - below surface level; ** surface elevation and coordinates are given for the Lokachi-5 borehole, which is a duplicate of the Lokachi-65 borehole

GAS SAMPLING SITES AND ANALYTICAL PROCEDURE

Four gas samples were collected from the producing boreholes. In the Lublin Basin, two gas samples come from the Middle Devonian reservoirs in the Ciecierzyn-1 (depth interval of 3740-3820 m) and Glinnik-3 (depth interval of 2293-2325 m) boreholes (Table 2). In the Lviv Basin, gas samples were taken from the Middle Devonian limestone and terrigenous rocks of the Lokachi-27 (depth interval of 815-870 m) and Lokachi-65 (depth interval of 938-956 m) boreholes. Free gas from the Lokachi-27, Lokachi-65 and Ciecierzyn-1 boreholes was sampled directly at the producing wellheads and put into metal containers $\left(\sim 1000 \mathrm{~cm}^{3}\right)$, while gas dissolved in oil in the Glinnik-3 borehole was taken from the separator to a glass container ( $500 \mathrm{~cm}^{3}$; Table 2).

The molecular compositions of the natural gases collected $\left(\mathrm{CH}_{4}, \mathrm{C}_{2} \mathrm{H}_{6}, \mathrm{C}_{3} \mathrm{H}_{8}, i \mathrm{C}_{4} \mathrm{H}_{10}, n \mathrm{C}_{4} \mathrm{H}_{10}, \mathrm{C}_{5} \mathrm{H}_{12}, \mathrm{C}_{6} \mathrm{H}_{14}, \mathrm{CO}_{2}, \mathrm{O}_{2}, \mathrm{H}_{2}\right.$, $\mathrm{N}_{2}, \mathrm{He}, \mathrm{Ar}$ ) were analysed with an Agilent $7890 \mathrm{~A}$ chromatograph (GC). The Agilent GC was equipped with a three-valve system using three $1 / 8$ inch packed columns $(3 \mathrm{ft}$ Hayesep Q 80/100 mesh, $6 \mathrm{ft}$ Hayesep Q 80/100 mesh and 10 $\mathrm{ft}$ molecular sieve $13 \times 45 / 60$ mesh) and a GS-Alumina capillary column $(50 \mathrm{~m} \times 0.53 \mathrm{~mm})$. The system consisted of two independent channels. The channel, which made use of FID for the detailed hydrocarbon analysis was a simple gas sampling valve injecting the sample into the GS-Alumina column. The second channel, involving packed columns, was used for determining methane, ethane and non-hydrocarbon gases. The GC oven was set as programmed: initial temperature $60^{\circ} \mathrm{C}$ held for $1 \mathrm{~min}$., then increased to $90^{\circ} \mathrm{C}$ at rate of $10^{\circ} \mathrm{C} / \mathrm{min}$., again increased to $190^{\circ} \mathrm{C}$ at rate of $20^{\circ} \mathrm{C} / \mathrm{min}$. and finally held for $5 \mathrm{~min}$. The front detector (TCD) was operated at a temperature of $150^{\circ} \mathrm{C}$ and the back detector (FID) at a temperature of $250^{\circ} \mathrm{C}$. Helium was used as a carrier gas with flow through the TCD channel of $28 \mathrm{ml} / \mathrm{min}$. and through the FID channel of $7 \mathrm{ml} / \mathrm{min}$. He concentration was determined on a Chrom- 5 GC equipped with TCD and $1 / 8$ in. $2.5 \mathrm{~m}$ long packed column with a mixture of $4 \mathrm{~A}$ and $5 \mathrm{~A}$ molecular sieves $(2: 1 \mathrm{v} / \mathrm{v})$ using argon as the carrier gas at a constant temperature of $25^{\circ} \mathrm{C}$. The Ar concentration was determined on the same $\mathrm{GC}$ at the same temperature program as helium using a $2.5 \mathrm{~m}$ long packed column filled with a $13 \mathrm{X}$ molecular sieve and hydrogen as carrier gas.

Stable isotope analyses were performed using Finnigan Delta Plus and Micromass VG Optima mass spectrometers. The stable carbon isotope data were expressed in the $\delta$-notation relative to VPDB on a scale such that NBS-22 (oil reference material) is $-30.03 \%$. The stable hydrogen isotope data were reported in delta notation $\left(\delta^{2} \mathrm{H}, \%\right)$ relative to the international standard, Vi- enna Standard Mean Ocean Water (VSMOW = 0.0\%o) and normalized to Standard Light Arctic Precipitation SLAP (2-point calibrations) as recommended by Coplen (2011). Analytical precision was estimated to be $\pm 0.2 \%$ for carbon and $\pm 3 \%$ o for hydrogen isotopes. Methane, ethane, propane, $i$-butane and $n$-butane were separated chromatographically for stable carbon isotope analyses. Water resulting from the combustion of methane for stable hydrogen isotope analyses was reduced to molecular hydrogen with zinc. The method was developed by Coleman et al. (1982) and we used its version modified by Florkowski (1985). The results of stable nitrogen isotope analyses were presented in $\delta$-notation $\left(\delta^{15} \mathrm{~N}, \%\right.$ ) relative to the air nitrogen standard. Analytical precision was estimated to be $\pm 0.4 \%$. Molecular nitrogen was separated chromatographically for stable nitrogen isotope analysis and transmitted to the mass spectrometer via the on-line system.

\section{RESULTS AND DISCUSSION}

\section{GEOCHEMICAL CHARACTERISTICS OF ORGANIC MATTER}

Previous geochemical studies reveal that the main source rocks in the Lviv and Lublin basins are Ordovician and Silurian strata (Kotarba et al., 2011; Więcław et al., 2011, 2012; Radkovets, 2015). The Middle and Upper Devonian, Mississippian, Middle and Upper Jurassic and Upper Cretaceous are of minor importance (Kotarba et al., 1998, 2003, 2011; Kosakowski et al., 2012a, b).

The hydrocarbon potential of the Lower Devonian in the Lublin Basin is generally poor to good (Table 1), and the TOC content ranges from 0.01 to $1.82 \mathrm{wt} . \%$ (median $0.06 \mathrm{wt} . \%$ ). In the Lochkovian the TOC varies generally from 0.01 to 0.70 wt. $\%$ with only one sample reaching $1.82 \mathrm{wt} \%$ in the Krowie Bagno IG 1. In the Emsian the TOC varies generally from 0.01 to $0.46 \mathrm{wt} . \%$ (median $0.06 \mathrm{wt} . \%$ ), with only one sample reaching 1.16 wt. \% in the Szwejki IG 3 borehole (Table 1 and Fig. 5). In the Lviv Basin, the hydrocarbon potential of the Lower Devonian is generally poor to fair (Table 1). The TOC content varies from 0.01 to $0.20 \mathrm{wt} . \%$ (median $0.04 \mathrm{wt} . \%$ ) in the Lochkovian and from 0.04 to $0.45 \mathrm{wt} \%$ (median $0.12 \mathrm{wt} \%$ ) in the Pragian-Emsian (Table 1 and Fig. 5). The Rock-Eval $T_{\max }$ values (Table 1) indicate that transformation of organic matter in the Lower Devonian varies from immature in the Lochkovian of the Lviv Basin to mature and overmature in the Emsian of the Lublin Basin (Table 1 and Fig. 6). Organic matter in the Lower Devonian contains mainly Type-II kerogen, and underwent primary and/or secondary oxidation processes (Figs. 6 and 7). 

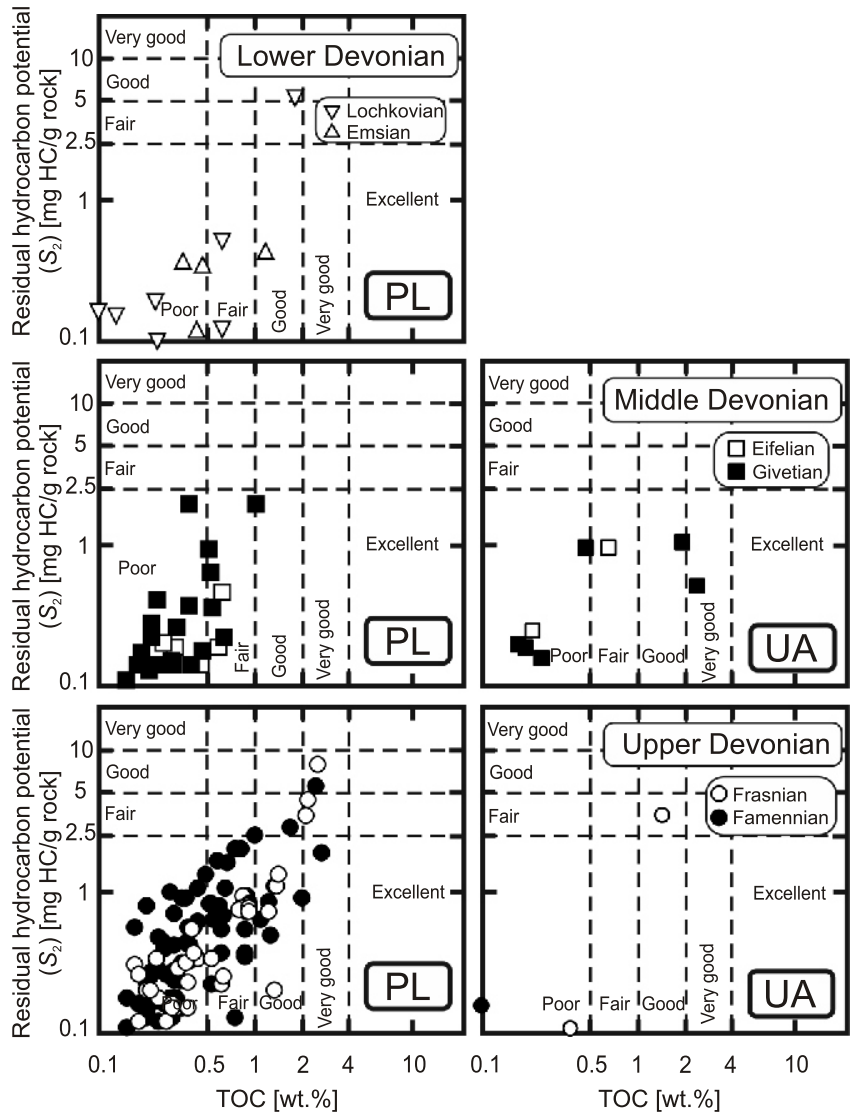

Fig. 5. Petroleum source quality of residual hydrocarbon potential versus total organic carbon (classification after Peters and Cassa, 1994)
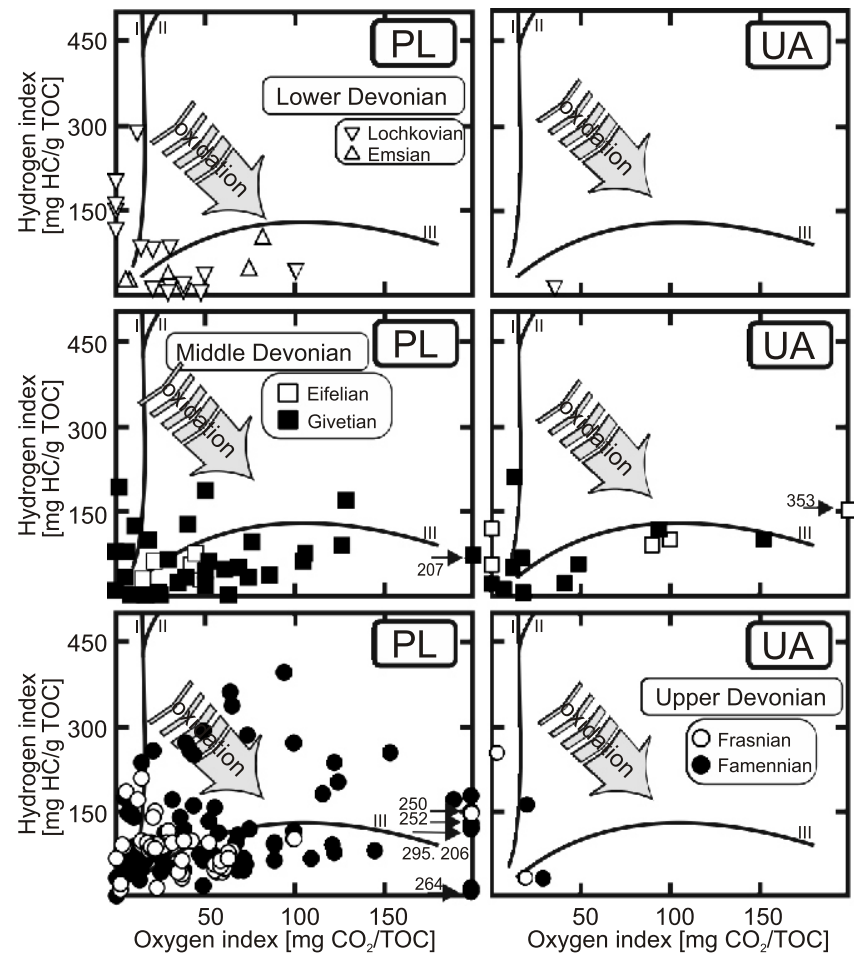

Fig. 7. Rock-Eval hydrogen index versus oxygen index (maturity paths of individual kerogen types after Espitalié et al., 1985)

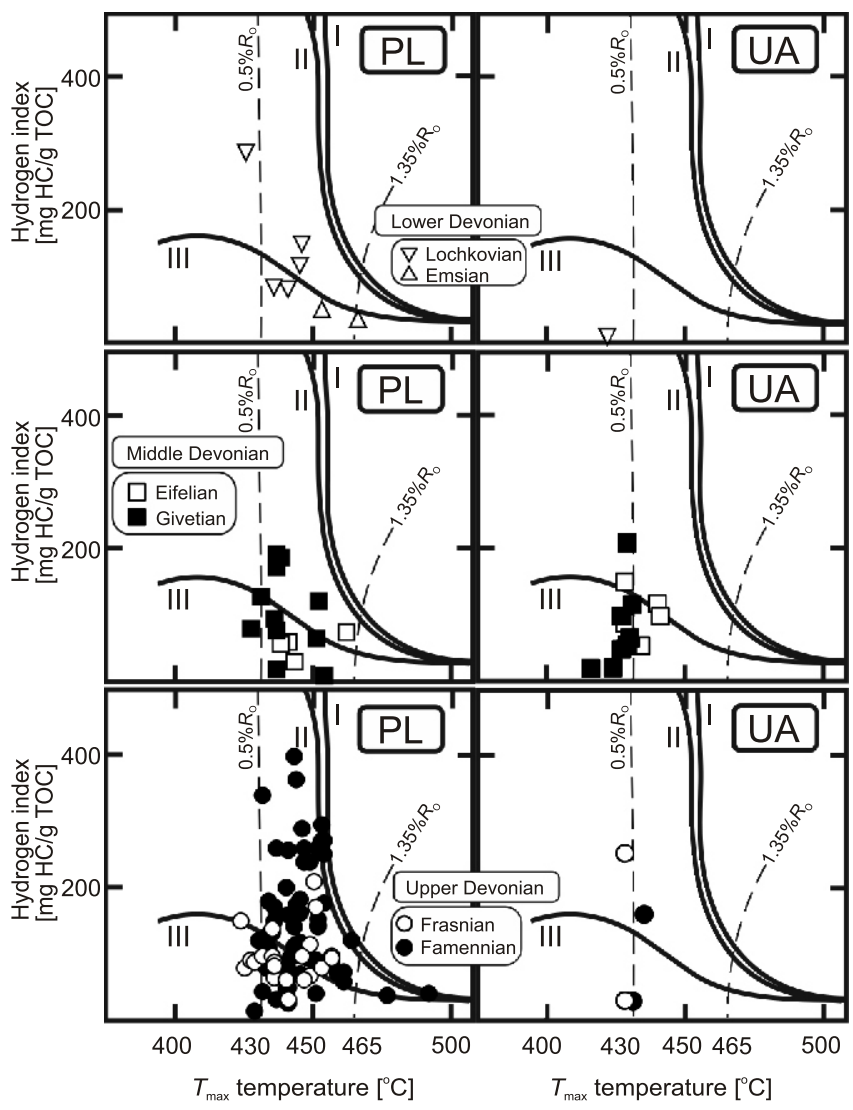

Fig. 6. Rock-Eval hydrogen index versus $T_{\max }$ temperature (genetic paths and maturity fields after Espitalié et al., 1985)

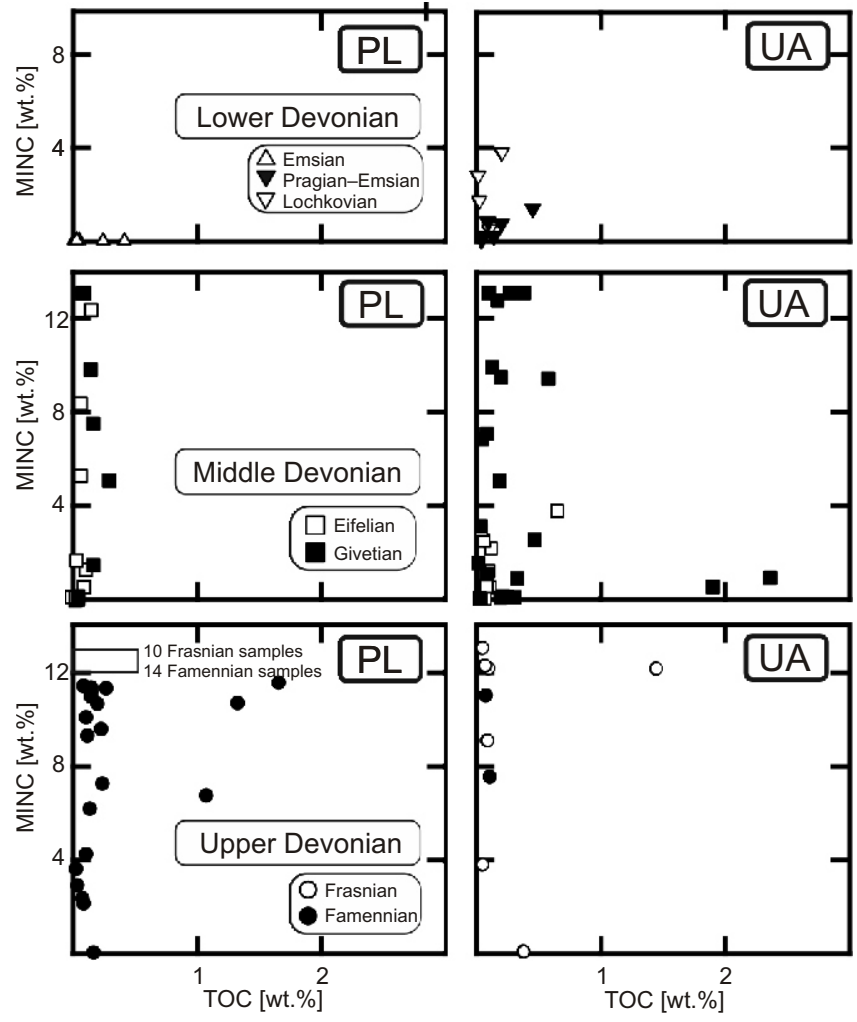

Fig. 8. Rock-Eval total organic carbon versus mineral carbon 
In the Middle Devonian of the Lublin Basin, the TOC content varies from 0.00 to 0.62 wt. $\%$ (median 0.16 wt. $\%$ ) in the Eifelian, and from 0.03 to $1.63 \mathrm{wt} . \%$ (median $0.18 \mathrm{wt} . \%$ ) in the Givetian (Table 1 and Fig. 5). The Rock-Eval $T_{\max }$ values indicate that organic matter is mature in the whole succession (Table 1 and Fig. 6). The hydrocarbon potential is generally poor and fair. In the Lviv Basin, the TOC content varies from 0.02 to 0.64 wt. $\%$ (median 0.08 wt.\%) in the Eifelian, and from 0.01 to 2.35 wt.\% (median 0.19 wt.\%) in the Givetian (Table 1 and Fig. 5). Rock-Eval $T_{\max }$ values indicate that transformation of organic matter is immature in the Givetian and mature in the Eifelian (Table 1 and Fig. 6). The hydrocarbon potential is from poor to good (Table 1 and Fig. 5).

In the Upper Devonian of the Lublin Basin, the TOC content varies from 0.02 to $2.44 \mathrm{wt} . \%$ (median $0.17 \mathrm{wt} \%$ ) in the Frasnian, and from 0.00 to $2.62 \mathrm{wt} . \%$ (median $0.18 \mathrm{wt} . \%$ ) in the Famennian (Table 1 and Fig. 5). The hydrocarbon potential is from poor to very good in the Frasnian and Famennian. The previous geochemical studies (Rock-Eval pyrolysis, $n$-alkane and isoprenoid distribution and stable carbon isotopes) of 198 samples from the Devonian of the Radom-Lublin area (Kotarba et al., 1998) reveal that fair source horizons occur within the Famennian Bychawa Formation having TOC from 0.1 to $1.25 \mathrm{wt} \%$, and low-temperature thermogenic transformation ("oil window") oil-prone Type-Il kerogen predominates. Worse source horizons occur within the Famennian Niedrzwica Formation (Kotarba et al., 1998). In the Lviv Basin, the TOC content varies from 0.04 to $1.43 \mathrm{wt} . \%$ (median $0.08 \mathrm{wt} . \%$ ) in the Frasnian, and from 0.07 to 0.10 wt. \% (median 0.09 wt. \%) in the Famennian (Table 1 and Fig. 5). Rock-Eval $T_{\max }$ values indicate that transformation of organic matter is mature in the Famennian, and overmature in the Frasnian (Table 1 and Fig. 6). The hydrocarbon potential is generally from poor to good in the Frasnian (Table 1 and Fig. 5).

Diagrams of hydrogen index versus $T_{\max }$ temperature (Fig. 6) and oxygen index (Fig. 7) suggest that mixed Type-II/III kerogen dominates in the Middle and Upper Devonian of the Lublin and Lviv basins. However, the shift in the direction of Type-III kerogen can be connected with the primary oxidation during sedimentation and/or secondary hydrothermal oxidation of dispersed organic matter during dolomitization (Fig. 7), indicating the domination of Type-II kerogen. These suggestions were confirmed by the results of sophisticated analyses (biomarker distribution, elemental composition of kerogen and stable carbon isotopes) from Kotarba et al. (1998, 2005).

Rock-Eval mineral (equivalent of carbonate) carbon content is insignificant in the Lower Devonian, and ranges from 0.01 to 3.76 wt. $\%$ with median values varying from 0.03 wt. $\%$ in the Emsian of the Lublin Basin, through 0.45 wt.\% in the Pragian-Emsian of the Lviv Basin, to $1.70 \mathrm{wt} \%$ in the Lochkovian of the Lviv Basin (Table 1 and Fig. 8). In the Middle and Upper Devonian, the parameter varies from 0.00 to 13.0 wt. $\%$, with median values ranging from 0.58 wt. $\%$ in the Eifelian of the Lublin Basin, through 0.89 wt.\% in the Eifelian and 3.12 wt.\% in the Givetian of the Lviv Basin, to 13.0 wt.\% in the Frasnian of the Lublin Basin (Table 1 and Fig. 8). The TOC content was observed to increase with the increasing of the carbonate (mineral carbon) content, mainly in the Eifelian and Givetian of the Lviv Basin, and in the Famennian of the Lublin Basin (Table 1 and Fig. 8).

\section{PETROGRAPHY OF RESERVOIR ROCKS}

Seven general types of reservoir rock can be distinguished in the Devonian of the Lublin and Lviv basins. These are sandstones, siltstones, dolomites, biodetrital dolomitized limestones, limestones, marls and anhydrites.
Sandstones form reservoir horizons in the Middle and Upper Devonian in the Lublin and Lviv basins. They are saturated in the Middle Devonian in the Lopushany, Pelcha, and Strutyn suites in the Lokachi field (Lviv Basin), and in the Telatyń Formation in the Komarów field (Lublin Basin). Thin intercalations of saturated sandstone occur also in the the Upper Devonian in the Lublin Basin - in the Frasnian Modryń Formation in the Mełgiew fields, and in the Famennian Hulcze Formation in the Glinnik and Ciecierzyn fields (Fig. 4). The Middle Devonian sandstones (Fig. 9A, B) are composed of semi-rounded and in places rounded grains, represented by quartz (80-90\%), seldom by feldspar (0.1-5\%), and muscovite scales (0.1-1\%; Appendix 2). Two kinds of matrix are observed: carbonate (4-19\%) - represented by dolomitised calcite (Fig. 9A) and clay (3-13\%) - composed of hydromica (Fig. 9B). Zircon is sporadically observed as an accessory mineral. The matrix is contact-porous and contact. Regardless of the matrix type, the pore space in the rocks is formed by intergranular spaces of 0.05 to $0.5 \mathrm{~mm}$ size (Fig. 9A-C). The porosity observed in the Middle Devonian sandstones ranges from 0.8 to $1.2 \%$ in the Eifelian in the Lublin Basin, and from 1 to $6.5 \%$ in the Givetian in the Lviv Basin (Appendix 2). According to Fedyshyn (1998), in the Lokachi field the porosity of sandstones increases up to $19.8 \%$, and permeability varies from 0.02 to $8.33 \mathrm{mD}$. The Upper Devonian sandstones are characterized by contact-porous and porous hydromica matrix, which reaches up to $21 \%$ of the rock. Intergranular pores in the rock are from 0.08 to $1.3 \mathrm{~mm}$ (Fig. 9D, E). The observed porosity of these sandstones ranges from 2.5 to $5.5 \%$ in the Famennian in the Lublin Basin (Apprendix 2). It increases up to $13.0 \%$ in the Glinnik field (Helcel-Weil and Dzięgielowski, 2003; Rzeźnik, 2012). The grains are represented by terrigenous quartz (up to $73 \%$ ), authigenic glauconite (locally up to $7 \%$ ), feldspar (4-6\%), and muscovite (1.5-2\%). Ore minerals are represented by pyrite (up to $5 \%$ ).

Siltstones form reservoir horizons in the same saturated horizons as sandstones. The Middle Devonian siltstones (Fig. 9C) are usually finely laminated. The fabric is formed by alternating streaks of clay material up to $0.5 \mathrm{~mm}$ thick and lens-like accumulations of silt, composed of quartz grains (60-67\%), feldspar (up to $3 \%$ ) rhombohedral dolomite (up to $5 \%$ ), muscovite scales ( $2-3 \%)$, glauconite (up to $1 \%$ ) and pyrite $(1-7 \%)$. Organic matter is present in a vein-like accumulations. The matrix is of porous and contact-porous type, represented by hydromica (up to $22 \%$ ) and a small admixture of dolomite (0.5-5\%). Intergranular pores of 0.05 to $0.3 \mathrm{~mm}$ size have been observed in the siltstones (Fig. 9C). The porosity observed in the Middle Devonian siltstones ranges from 7 to $8.5 \%$ in the Lviv Basin (Apprendix 2).

Dolomites form reservoir horizons in the Middle Devonian in the Lokachi field in the Lviv Basin, as well as in the Middle Devonian in the Komarów field, and in the Frasnian in the Ciecierzyn and Mełgiew fields in the Lublin Basin. The Middle Devonian dolomites contain 92 to $97 \%$ of $\mathrm{CaMg}\left(\mathrm{CO}_{3}\right)_{2}$ and $1-4 \%$ of clay minerals. The presence of pyritised streaks and organic matter gives a grey and dark grey colour to these rocks. The dolomites (Fig. 10B, C) are composed of microcrystalline and fine- to medium-grained $(0.3-2 \mathrm{~mm})$ dolomite crystals, that usually have rhombohedral shape and developed intergranular porosity. The pore space is represented mainly by isometric-irregular pores and fracture-like micro-cavities of 0.01 to $0.5 \mathrm{~mm}$ size. The observed porosity in the Middle Devonian dolomites ranges from 3.0 to $9.0 \%$ in the Givetian in the Lviv Basin (Appendix 2). According to Fedyshyn (1998), dolomites in the Lokachi field have the pore and fracture-type porosity varying from 3 to $16.5 \%$, and permeability from 0.01 to $4.34 \mathrm{mD}$. The Upper Devonian dolomites are usually represented by fine- to medium-grained dolomite crystals of rhombohedral shape with intergranular po- 

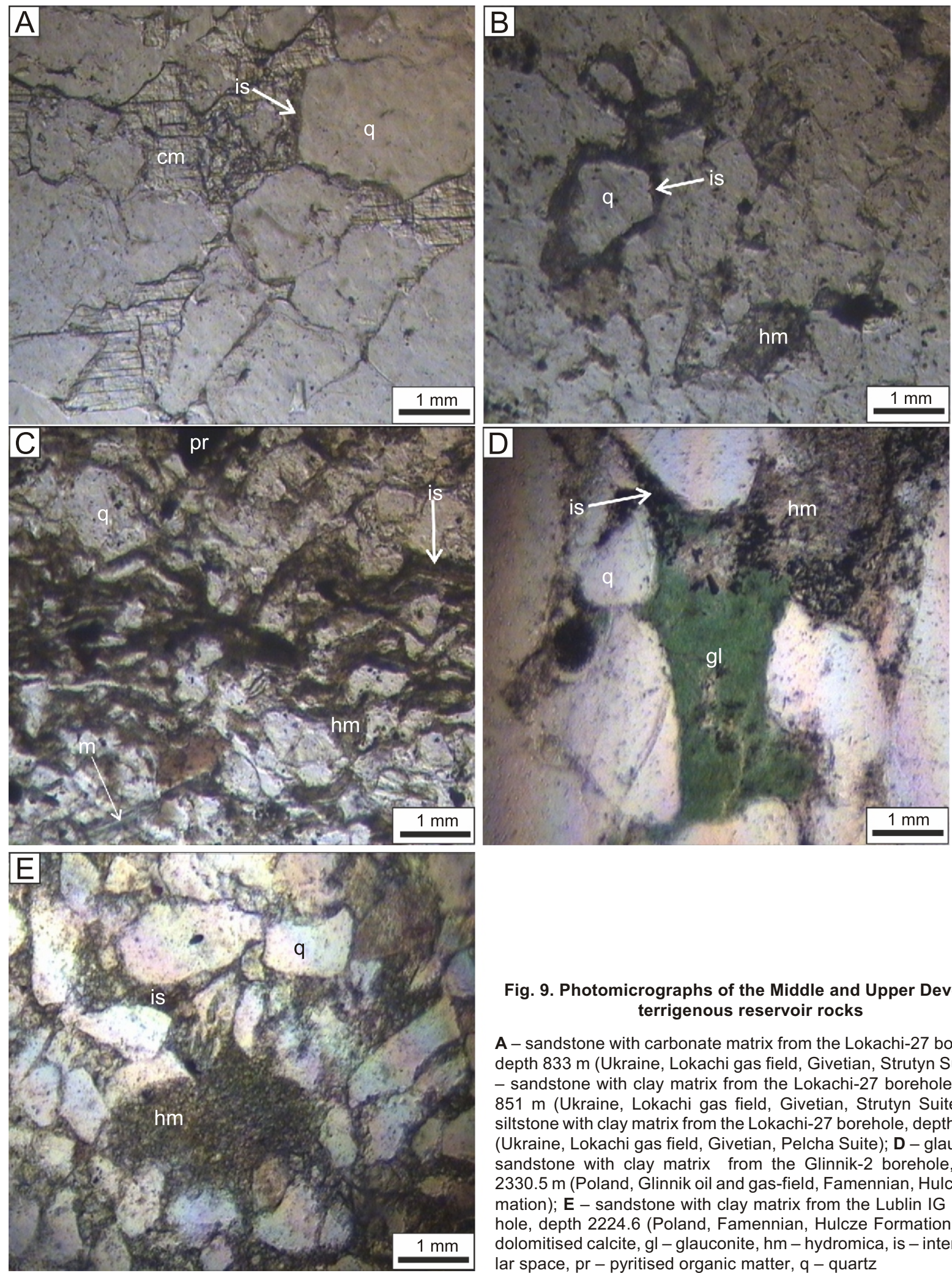

Fig. 9. Photomicrographs of the Middle and Upper Devonian terrigenous reservoir rocks

A - sandstone with carbonate matrix from the Lokachi-27 borehole, depth 833 m (Ukraine, Lokachi gas field, Givetian, Strutyn Suite); B - sandstone with clay matrix from the Lokachi-27 borehole, depth 851 m (Ukraine, Lokachi gas field, Givetian, Strutyn Suite); C siltstone with clay matrix from the Lokachi-27 borehole, depth $863 \mathrm{~m}$ (Ukraine, Lokachi gas field, Givetian, Pelcha Suite); D - glauconitic sandstone with clay matrix from the Glinnik-2 borehole, depth $2330.5 \mathrm{~m}$ (Poland, Glinnik oil and gas-field, Famennian, Hulcze Formation); $\mathbf{E}$ - sandstone with clay matrix from the Lublin IG 1 borehole, depth 2224.6 (Poland, Famennian, Hulcze Formation); $\mathrm{cm}$ dolomitised calcite, $\mathrm{gl}$ - glauconite, $\mathrm{hm}$ - hydromica, is - intergranular space, $\mathrm{pr}$ - pyritised organic matter, q - quartz 

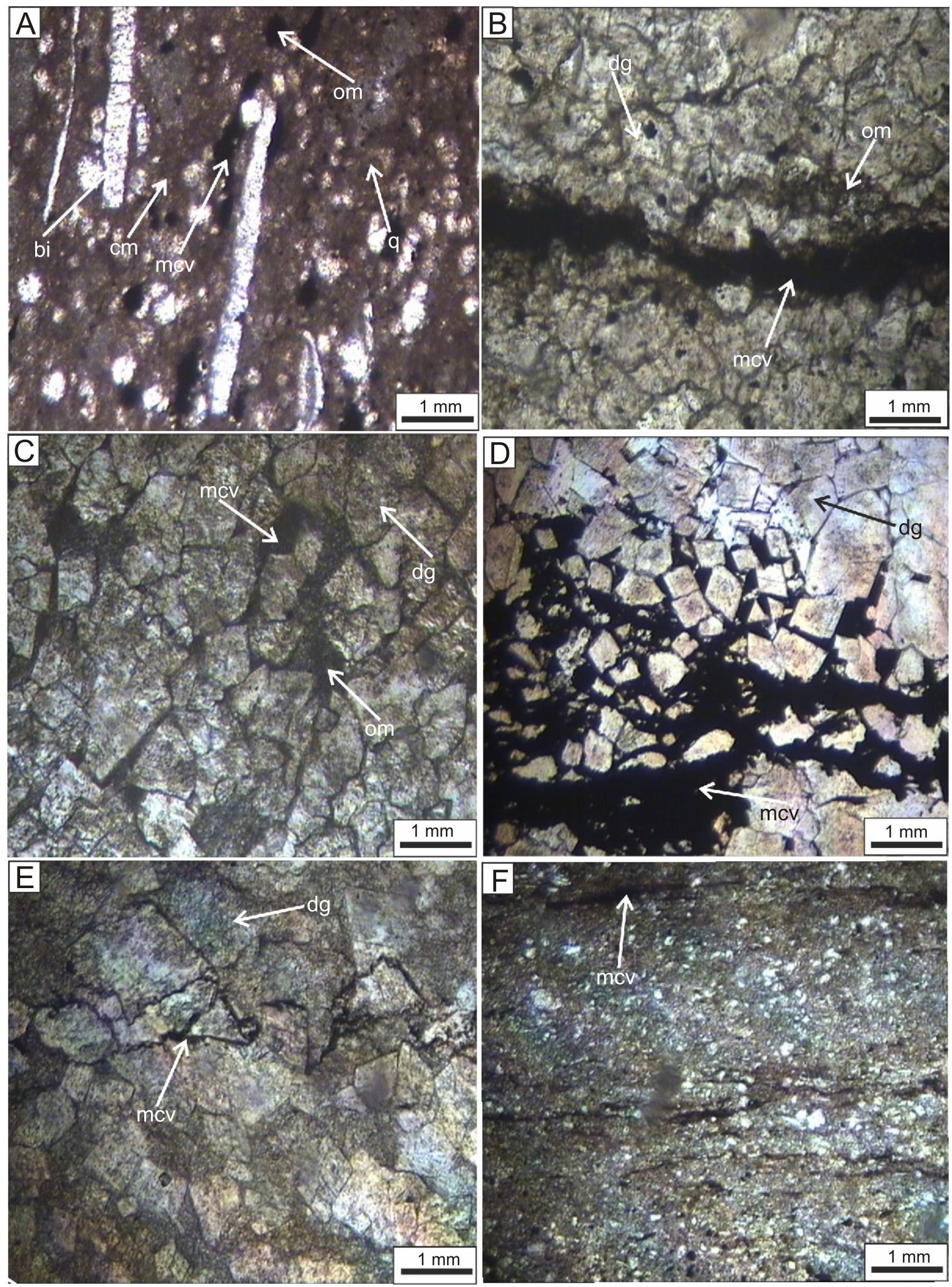

Fig. 10. Photomicrographs of the Middle and Upper Devonian carbonate reservoir rocks

A - biodetrital dolomitised limestone from the Lokachi-27 borehole, depth $816 \mathrm{~m}$ (Ukraine, Lokachi gas field, Givetian, Strutyn Suite); B-E fine- to medium-grained dolomite: B - from the Lokachi-5 borehole, depth $905.6 \mathrm{~m}$ (Ukraine, Lokachi gas field, Givetian, Pelcha Suite), C from borehole Lokachi-27, depth $832 \mathrm{~m}$ (Ukraine, Lokachi gas field, Givetian, Strutyn Suite), D - from the Ciecierzyn-1 borehole, depth $3776.5 \mathrm{~m}$ (Poland,Ciecierzyn oil field, Famennian, Bychawa Formation), E - from the Lublin IG 1 borehole, depth $4234 \mathrm{~m}$ (Poland, Famennian, Bychawa Formation); F - marl from the Lublin IG 1 borehole, depth 3512.6 m (Poland, Frasnian, Mordyń Formation); bi - calcareous bioclasts, dg - dolomite graine, mcv - micro-cavities, om - organic matter, other explanations as in Figure 9 

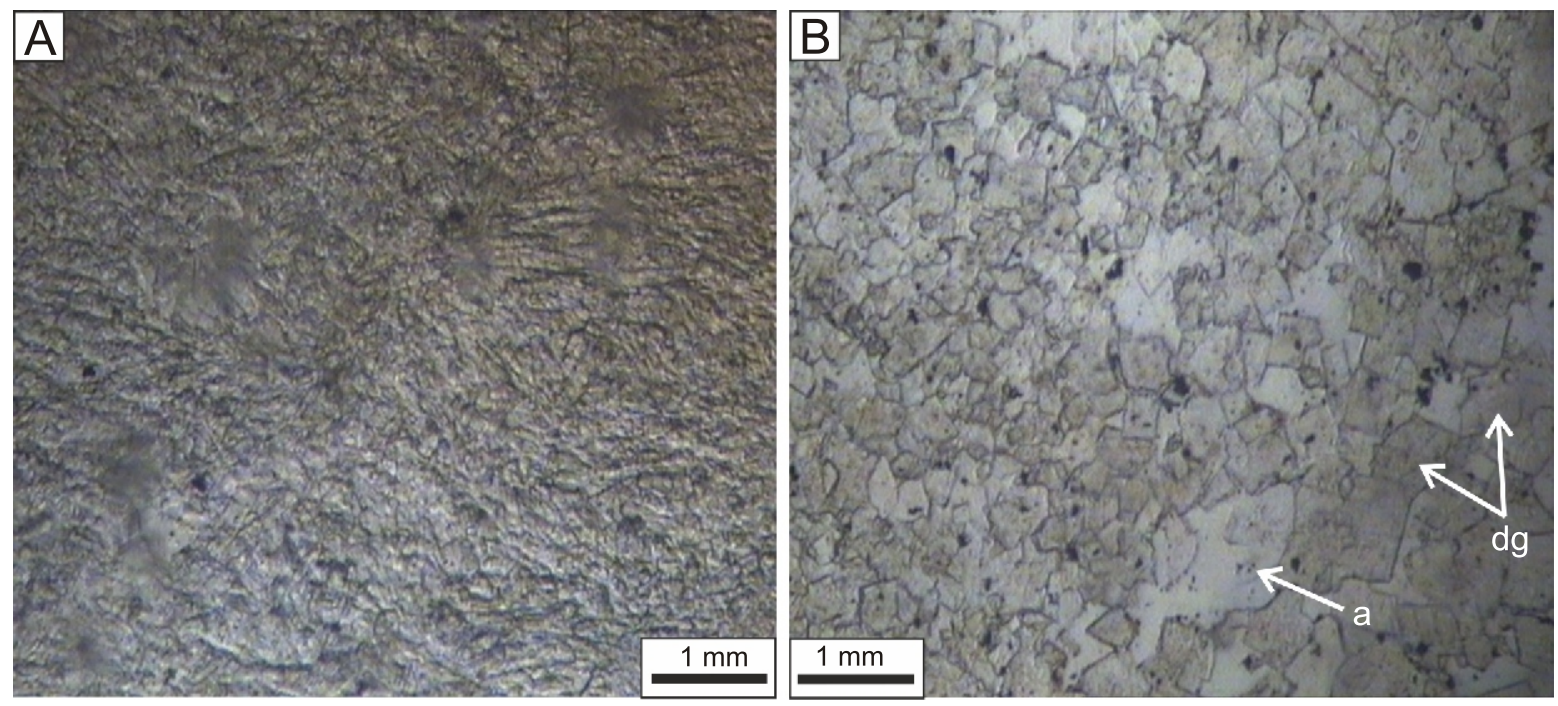

Fig. 11. Photomicrographs of the Middle Devonian sulphate and carbonate-sulphate sealing rocks from the Lokachi gas field

A - anhydrite (Lokachi-27, depth $827.8 \mathrm{~m}$ ); B - dolomite with anhydrite concretions (Lokachi-5, depth $841.9 \mathrm{~m}$ ); a - anhydrite, other explanation as in Figure 10

rosity developed and fracture-like micro-cavities 0.01 to $0.5 \mathrm{~mm}$ in size (Fig. 10D, E). Pores are filled with microcrystalline pyrite the amount of which reaches $5 \%$ of the rock. The observed porosity in the Upper Devonian dolomites ranges from 1.5 to $4.5 \%$ in the Frasnian in the Lviv Basin, and from 0.5 to $1.5 \%$ in the Frasnian, and from 0.5 to $2.5 \%$ in the Famennian in the Lublin Basin (Appendix 2). In the Ciecierzyn and Metgiew A fields, the dolomites have fracture-type porosity from 1.0 to $2.5 \%$, and permeability estimated at $0.1 \mathrm{mD}$ (Stachurski et al., 1985 Modzelewski, 1999; Helcel-Weil and Dzięgielowski, 2003; Helcel-Weil et al., 2007).

Biodetrital dolomitised limestones (Fig. 10A) form reservoir horizons in the Middle Devonian in the Lokachi field in the Lviv Basin. These limestones are composed of skeletal debris $(35 \%)$ with brachiopods, tentaculites, and fewer corals and calcareous bioclasts. A small admixture of pyrite and organic matter, which fill the lenticular micro-cavities, is also observed. The matrix is composed of microcrystalline to fine-crystalline carbonates with $69-76 \%$ calcite, $23-26 \%$ dolomite, and $1-3 \%$ clay minerals. The porosity of these limestones ranges from 0.7 to 2.5\% (Appendix 2).

Limestones form reservoir horizons in the Upper Devonian in the Mełgiew B field in the Lviv Basin. According to Helcel-Weil and Dzięgielowski (2003) these limestones have fracture-type porosity up to $1.4 \%$.

Marls (Fig. 10F) form reservoir horizons in the Upper Devonian Bychawa Formation in the Ciecierzyn field (Lublin Basin) The marls are composed of $44-48 \%$ calcite, $38-46 \%$ clay minerals and $7-12 \%$ dolomite. Thick micro-cavities up to $0.1 \mathrm{~mm}$ filled with organic matter and pyrite (up to 5\%) are observed. The porosity of these marls ranges from 0.7 to $2.5 \%$ in the Famennian in the Lublin Basin (Appendix 2).

Anhydrites (Fig. 11A) and dolomites with anhydrite concretions (Fig. 11B) form a reservoir horizon in the Telatyń Formation in the Komarów field. The anhydrites are composed of fineand medium-grained parallel-oriented or tangled-fibrous aggregates of anhydrite crystals (1-2\%) with clay films $>0.1 \mathrm{~mm}$ occuring at the bedding planes. The dolomites are composed of fine rhombohedral grains of dolomite $(0.1-1 \mathrm{~mm})$, among which the irregular crystals of anhydrite are observed. Pyrite aggre- gates (up to $0.1 \mathrm{~mm}$ ) are always present. The filtration properities in these rocks are related to secondary fractures and caverns with no primary porosity (Appendix 2).

ORIGIN OF NATURAL GAS

The gas analysed, collected from the Middle and Upper Devonian reservoirs (Fig. 1 and Table 2), shows variation in molecular and isotopic composition. The molecular composition and indices, and isotopic composition are reported in Tables 3 and 4 , respectively.

Hydrocarbon gas. For classification of the hydrocarbon gas analysed, the diagnostic diagrams (Figs. 12 and 13) were applied after Whiticar et al. (1986), Schoell (1988), Whiticar (1994) and Berner and Faber (1996, 1997). An important implication from the interpretation is that a linear relationship of stable carbon isotopes of methane, ethane, propane and butanes versus their reciprocal carbon number (Fig. 14) as assumed by Chung et al. (1988) and Rooney et al. (1995) is not a sufficient indicator of natural gas generated from a single source. Zou et al. (2007) and Kotarba et al. (2009) suggested that in this type of plot a "dogleg" trend, characterized by relatively ${ }^{13} \mathrm{C}$-depleted methane and ${ }^{13} \mathrm{C}$-enriched propane compared to ethane, is indicative of natural gas that was not generated from a single source rock (multiple source) or that has undergone post-generation alteration (e.g., secondary gas cracking, microbial oxidation, thermochemical sulphate reduction). Moreover, the degree of ${ }^{13} \mathrm{C}$ depletion of methane in relation to ethane can be used for evaluating the mixing proportion of microbial methane and thermogenic gas (Kotarba and Lewan, 2004; Kotarba et al., 2009).

The plots of stable carbon isotope composition of methane versus hydrocarbon index $\mathrm{C}_{\mathrm{HC}}$ (Fig. 12A) and hydrogen isotope compositions of methane (Fig. 12B) from natural gas accumulations in the Upper Devonian Ciecierzyn and Glinnik (Cn-1 and GI-3 samples) reservoirs (Fig. 4A, B) indicate that this gas was generated mainly during a low-temperature thermogenic process. The gases from the Middle Devonian Lokachi (Lo-27 and Lo-65 samples; Fig. 4C) reservoirs contain a significant component of microbial methane. Microbial ethane enriched in ${ }^{12} \mathrm{C}$ 
Molecular composition of analysed natural gases

\begin{tabular}{|l|c|c|c|c|c|c|c|c|c|c|c|c|c|}
\hline $\begin{array}{c}\text { Sample } \\
\text { code }\end{array}$ & $\mathrm{CH}_{4}$ & $\mathrm{C}_{2} \mathrm{H}_{6}$ & $\mathrm{C}_{3} \mathrm{H}_{8}$ & $i \mathrm{C}_{4} \mathrm{H}_{10}$ & $n \mathrm{C}_{4} \mathrm{H}_{10}$ & $i \mathrm{C}_{5} \mathrm{H}_{12}$ & $n \mathrm{C}_{5} \mathrm{H}_{12}$ & $\mathrm{C}_{6} \mathrm{H}_{14}$ & $\mathrm{~N}_{2}$ & $\mathrm{CO}_{2}$ & $\mathrm{He}$ & $\mathrm{Ar}$ & $\mathrm{H}_{2}$ \\
\hline \multicolumn{10}{|c|}{ Lviv Basin } \\
\hline Lo-27 & 927 & 1.55 & 0.24 & 0.10 & 0.09 & 0.10 & 0.07 & 0.01 & 4.79 & 0.03 & 0.25 & n.a. & 0.012 \\
\hline Lo-65 & 95.4 & 0.96 & 0.19 & 0.07 & 0.07 & 0.08 & 0.07 & 0.01 & 2.92 & 0.04 & 0.13 & n.a. & 0.012 \\
\hline \multicolumn{10}{|c|}{ Lublin Basin } \\
\hline Cn-1 & 92.1 & 3.80 & 0.80 & 0.08 & 0.11 & 0.03 & 0.03 & 0.02 & 2.70 & 0.20 & 0.10 & 0.004 & 0.004 \\
\hline Gl-3 & 83.2 & 7.82 & 3.93 & 0.41 & 1.17 & 0.11 & 0.30 & 0.10 & 2.52 & 0.20 & 0.25 & 0.007 & 0.003 \\
\hline
\end{tabular}

n.a. - not analysed

Table 4

Molecular indices and stable carbon, hydrogen and nitrogen isotope composition of the natural gas analysed

\begin{tabular}{|c|c|c|c|c|c|c|c|c|c|c|c|c|c|c|}
\hline \multirow{2}{*}{$\begin{array}{l}\text { Sample } \\
\text { code }\end{array}$} & \multicolumn{6}{|c|}{ Molecular indices } & \multicolumn{8}{|c|}{ Stable isotopes [\%o] } \\
\hline & $\mathrm{C}_{\mathrm{HC}}$ & $\mathrm{C}_{1} / \mathrm{C}_{2}$ & $\mathrm{C}_{2} / \mathrm{C}_{3}$ & $\mathrm{C}_{3} / \mathrm{C}_{4+}$ & $\begin{array}{l}i-\mathrm{C}_{4} / \\
n-\mathrm{C}_{4}\end{array}$ & $\begin{array}{l}i-\mathrm{C}_{5} / \\
n-\mathrm{C}_{5}\end{array}$ & $\begin{array}{c}\delta^{13} \mathrm{C} \\
\left(\mathrm{CH}_{4}\right)\end{array}$ & $\begin{array}{l}\delta^{2} \mathrm{H} \\
\left(\mathrm{CH}_{4}\right)\end{array}$ & $\begin{array}{l}\delta^{13} \mathrm{C} \\
\left(\mathrm{C}_{2} \mathrm{H}_{6}\right)\end{array}$ & $\begin{array}{l}\delta^{13} \mathrm{C} \\
\left(\mathrm{C}_{3} \mathrm{H}_{8}\right)\end{array}$ & $\begin{array}{c}\delta^{13} \mathrm{C} \\
\left(i \mathrm{C}_{4} \mathrm{H}_{10}\right)\end{array}$ & $\begin{array}{c}\delta^{13} \mathrm{C} \\
\left(n \mathrm{C}_{4} \mathrm{H}_{10}\right)\end{array}$ & $\begin{array}{l}\delta^{13} \mathrm{C} \\
\left(\mathrm{CO}_{2}\right)\end{array}$ & $\begin{array}{l}\delta^{15} \mathrm{~N} \\
\left(\mathrm{~N}_{2}\right)\end{array}$ \\
\hline \multicolumn{15}{|c|}{ Lviv Basin } \\
\hline Lo-27 & 52 & 59.7 & 6.4 & 0.6 & 1.2 & 1.37 & -48.0 & -181 & -33.3 & -27.1 & -26.2 & -26.3 & -8.1 & -8.2 \\
\hline Lo-65 & 83 & 99.8 & 5.0 & 0.7 & 0.9 & 1.17 & -50.2 & -194 & -32.3 & -26.7 & -27.0 & -27.2 & -8.9 & -7.9 \\
\hline \multicolumn{15}{|c|}{ Lublin Basin } \\
\hline $\mathrm{Cn}-1$ & 20 & 24.2 & 4.8 & 3.0 & 0.7 & 0.88 & -36.7 & -144 & -35.2 & -32.0 & n.a. & n.a. & -9.2 & -7.0 \\
\hline Gl-3 & 7 & 10.6 & 2.0 & 1.9 & 0.4 & 0.37 & -49.0 & -197 & -33.5 & -30.2 & n.a. & n.a. & n.a. & -11.3 \\
\hline
\end{tabular}

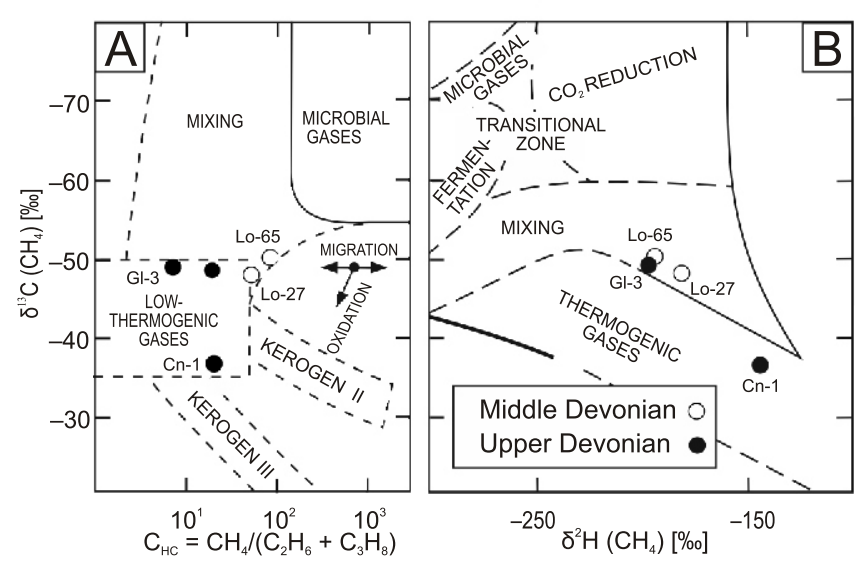

Fig. 12. $\delta^{13} \mathrm{C}$ of methane versus (A) hydrocarbon index $\mathrm{C}_{\mathrm{HC}}$ (i.e., $\left.\mathrm{CH}_{4} /\left[\mathrm{C}_{2} \mathrm{H}_{6}+\mathrm{C}_{3} \mathrm{H}_{8}\right]\right)$ and $(\mathrm{B}) \delta^{2} \mathrm{H}\left(\mathrm{CH}_{4}\right)$ for the natural gas accumulated in the Middle and Upper Devonian reservoirs of the study area

Genetic fields after Bernard et al. (1976) and Whiticar (1994); key for gas sample codes see Table 3 $\left(\delta^{13} \mathrm{C}\right.$ from -61 to $-52 \%$ ) has been reported in producing microbial gas accumulations (Lillis, 2007) and microbial propane in some deep marine deposits (Hinrichs et al., 2006). However, no microbial ethane and propane occur in such gas (Figs. 13, 14 and $15 \mathrm{E}, \mathrm{F}$ ). The stable carbon isotope compositions of ethane, propane and butanes (Figs. 13 and 14) suggest that the hydrocarbon thermogenic components were generated from Ordovician-Silurian Type-II kerogen (Kotarba et al., 2011; Więcław et al., 2011, 2012) and partly from Middle Devonian mixed Type-II/III kerogen (Figs. 13 and 14) of maturity from $\sim 0.9$ to $1.4 \%$ on the vitrinite reflectance scale (Fig. 14).

Carbon dioxide. Carbon dioxide can be produced under certain geological conditions as a result of various biogenic and abiogenic processes: microbial fermentation, thermogenic decomposition of organic matter, decarboxylation of lipids, bacterial respiration, hydrocarbon oxidation by thermochemical or microbial sulphate reduction and by mineralised waters, thermal decomposition of carbonate rocks, mantle degassing and carbonate reservoir dissolution by acid fluids (e.g., Gutsalo and Plotnikov, 1981; Kotarba, 1988, 2012; Jenden et al., 1993; Dai et al., 1996; Kotarba and Rice, 2001; Fischer et al., 2006; Zhang et al., 2008, and references therein). It has been observed that partial pressures of carbon dioxide increase systematically with increasing temperature in petroleum basins (Smith and Ehrenberg, 1989). In addition, this effect can be buffered by feldspars, clay minerals, or carbonates, and suggests that organically derived carbon dioxide may be removed from natural gas by mineral precipitation (Smith and Ehrenberg, 1989; Hutcheon and Abercrombie, 1990).

Carbon dioxide occurs in the natural gas analysed in concentrations from 0.03 to 0.20 vol. $\%$ (Table 3$)$ and $\delta^{13} \mathrm{C}\left(\mathrm{CO}_{2}\right)$ val- 


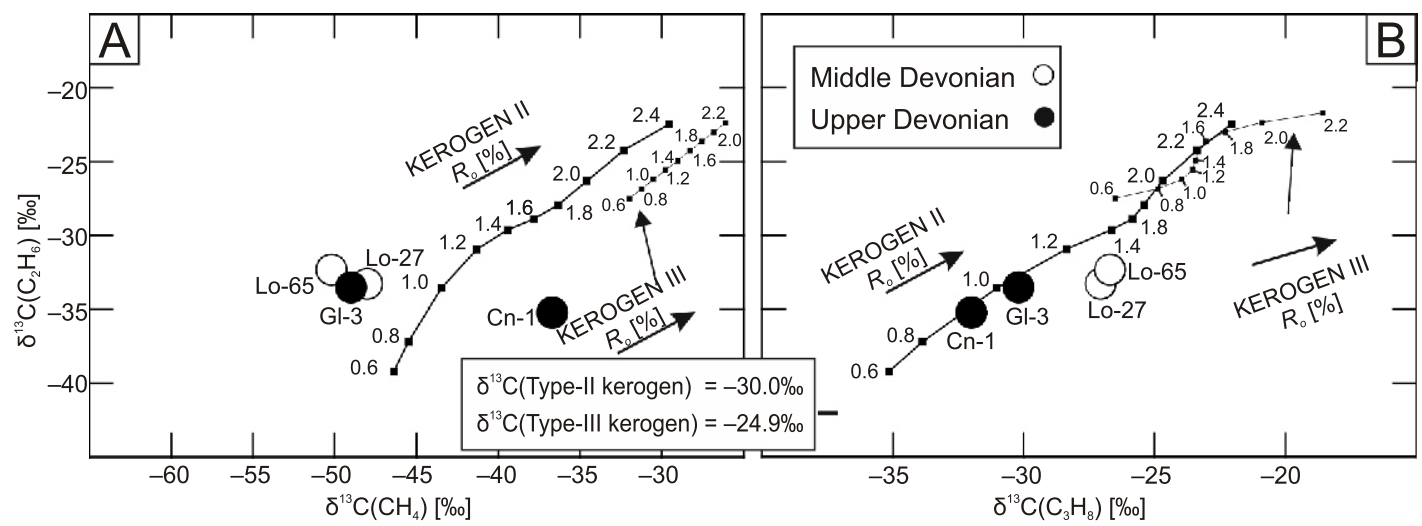

Fig. 13. $\delta^{13} \mathrm{C}\left(\mathrm{C}_{2} \mathrm{H}_{6}\right)$ versus $(\mathrm{A}) \delta^{13} \mathrm{C}\left(\mathrm{CH}_{4}\right)$ and $(\mathrm{B}) \delta^{13} \mathrm{C}\left(\mathrm{C}_{3} \mathrm{H}_{8}\right)$ for natural gas accumulated in the Middle and Upper Devonian reservoirs of the study area

Position of vitrinite reflectance curves for Type-II and -III kerogens after Berner and Faber (1996, 1997); curves were shifted based on average $\delta^{13} \mathrm{C}=-30.0 \%$ for Ordovician, Silurian, Upper and Middle Devonian Type-II kerogen after Kotarba et al. (1998) and Więcław et al. $(2011,2012)$, and average $\delta^{13} \mathrm{C}$ values $=-24.9 \%$ for Mississippian (clastic) Type-III kerogen after Więcław et al. (2011); key for gas sample codes see Table 3

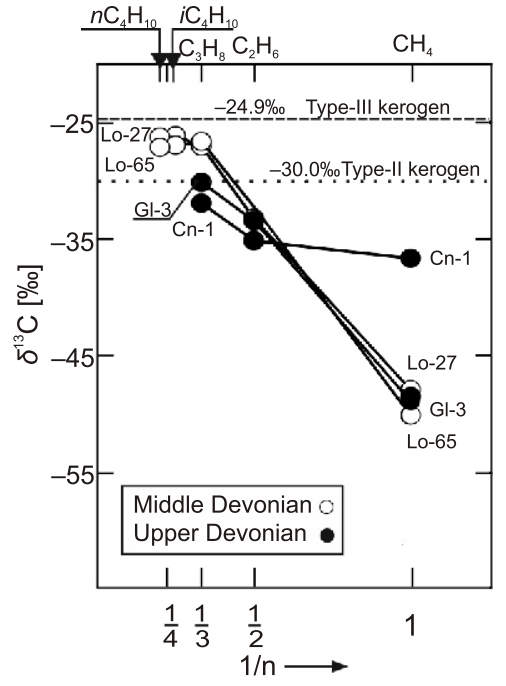

Fig. 14. Stable carbon isotope composition of methane, ethane, propane $i$-butane and $n$-butane versus the reciprocal of their carbon number for natural gas accumulated in the Middle and Upper Devonian reservoirs of the study area

Structure of the graph after Chung et al. (1988) and Rooney et al (1995); average values of $\delta^{13} \mathrm{C}=-30.0 \%$ for Ordovician, Silurian, Middle and Upper Devonian Type-II kerogen after Kotarba et al (1998) and Więcław et al. $(2011,2012)$, and average $\delta^{13} \mathrm{C}$ values $=$ -24.9\% Mississippian (clastic) Type-III kerogen after Więcław et al (2011); key for gas sample codes see Table 3 ues vary from -9.2 to $-8.1 \%$ (Table 4$)$. The insignificant carbon dioxide concentrations (Table 4 ), the values of the carbon dioxide-methane (CDMI) index (Fig. 15B) and plot of $\delta^{13} \mathrm{C}\left(\mathrm{CO}_{2}\right)$ versus $\delta^{13} \mathrm{C}\left(\mathrm{CH}_{4}\right)$ (Fig. 16) suggest that in the gas analysed from Ciecierzyn (Cn-1) a thermogenic component occurs, and in Lokachi (Lo-27 and Lo-65) both thermogenic and microbial components can appear together.

Molecular nitrogen. Molecular nitrogen is produced in large quantities during both microbial processes and the thermogenic transformation of organic matter (Kotarba, 1988; Krooss et al., 1995). The process of molecular nitrogen generation from organic matter was also documented by pyrolytic experiments (Gerling et al., 1997; Kotarba and Lewan, 2013). Molecular nitrogen can also released from $\mathrm{NH}_{4}$-rich illites that have undergone intense fluid/rock interaction (Mingram et al., 2005; Lüders et al., 2005). The $\delta^{15} \mathrm{~N}$-values of molecular nitrogen from natural gas vary from -15 to $18 \%$ o (Gerling et al., 1997). This isotopic fractionation results from both primary genetic factors and secondary processes taking place during gas migration through the gas-rock and gas-reservoir fluids interfaces (Stahl, 1977; Littke et al., 1995; Gerling et al., 1997; Zhu et al., 2000; Krooss et al., 2005; Mingram et al., 2005; Lüders et al., 2005).

Molecular nitrogen occurs in the natural gas analysed in concentrations from 2.52 to $4.79 \%$ (Table 3 ) and $\delta^{15} \mathrm{~N}\left(\mathrm{~N}_{2}\right)$ values vary from -11.3 to $-7.0 \%$ (Table 4 ). The generally increasing trend of $\delta^{15} \mathrm{~N}\left(\mathrm{~N}_{2}\right)$ values with the growth of $\mathrm{N}_{2}$ concentration (Fig. 17) may suggest that molecular nitrogen from the natural gas analysed was mainly generated during thermal transformation of organic matter, and in the cases of Ciecierzyn (Cn-1) and Lokachi (Lo-27 and Lo-65) gas (Fig. 17) insignificant volumes of it can also originate during destruction of $\mathrm{NH}_{4}$-rich illites of the clayey facies of the Ordovocian-Silurian strata. 

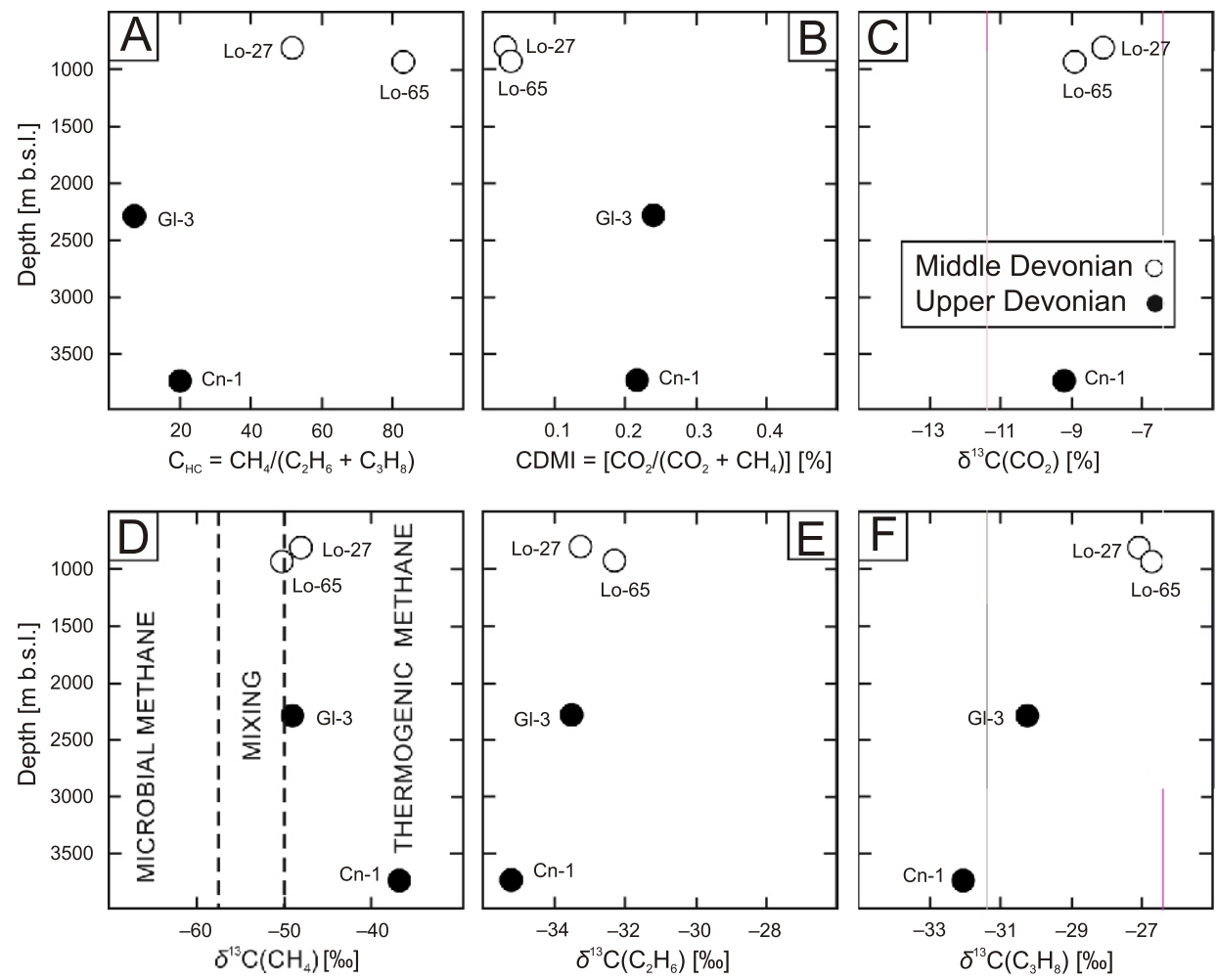

Fig. 15A - hydrocarbon index; $\mathrm{B}$ - carbon dioxide-methane index; $\mathrm{C}-\delta^{13} \mathrm{C}\left(\mathrm{CO}_{2}\right)$; $D-\delta^{13} \mathbf{C}\left(\mathrm{CH}_{4}\right) ; \mathrm{E}-\delta^{13} \mathrm{C}\left(\mathrm{C}_{2} \mathrm{H}_{6}\right) ; \mathrm{F}-\delta^{13} \mathrm{C}\left(\mathrm{C}_{3} \mathrm{H}_{8}\right)$ versus depth of natural gas accumulated in the Middle and Upper Devonian reservoirs of the study area

Key for gas sample codes see Table 3

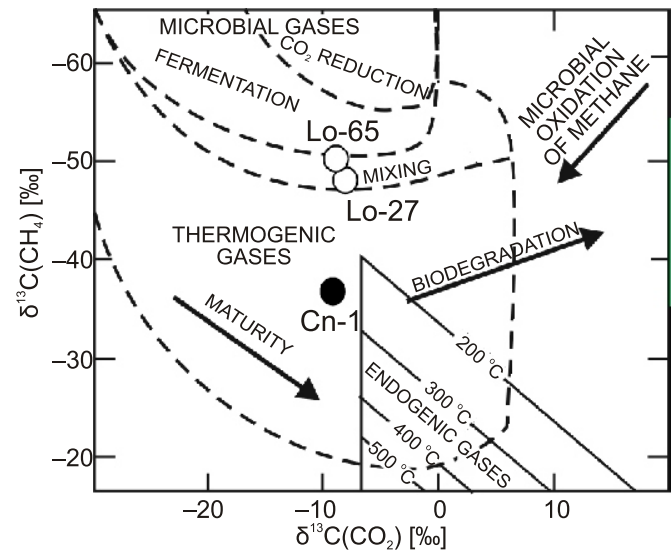

Fig. 16. $\delta^{13} \mathrm{C}\left(\mathrm{CH}_{4}\right)$ versus $\delta^{13} \mathrm{C}\left(\mathrm{CO}_{2}\right)$ for natural gas accumulated in the Middle and Upper Devonian reservoirs of the study area

Compositional genetic fields modified after Gutsalo and Plotnikov (1981), Milkov (2011) and Kotarba (2012); key for gas sample codes see Table 3

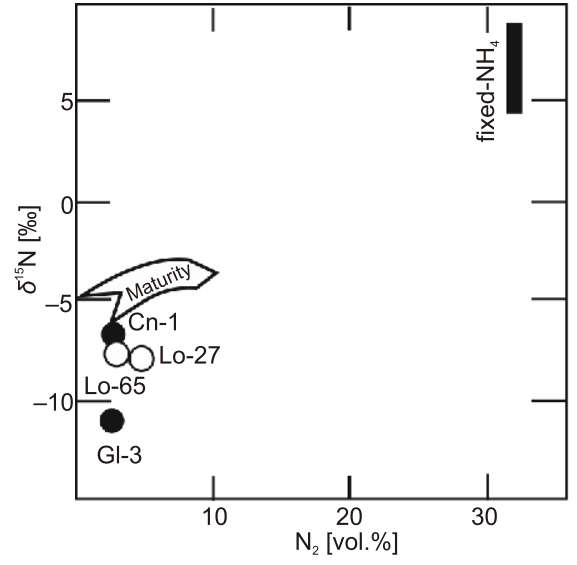

Fig. 17. $\delta^{15} \mathbf{N}\left(\mathbf{N}_{2}\right)$ versus $\mathbf{N}_{2}$ concentration of natural gas accumulated in the Middle and Upper Devonian reservoirs of the study area

Direction of increasing source rock after Gerling et al. (1997) and $\delta^{115} \mathrm{~N}$ range of fixed- $\mathrm{NH}_{4}$ in rich illites after Mingram et al. (2005); key for gas sample codes see Table 3 


\section{CONCLUSIONS}

1. In the Lower Devonian the TOC content ranges from 0.01 to $1.82 \mathrm{wt} . \%$ (median $0.06 \mathrm{wt} . \%$ ) in the Lublin Basin, and from 0.01 to 0.45 wt. \% (median 0.08 wt. \%) in the Lviv Basin. The organic matter contains mainly Type-II kerogen, and underwent primary and/or secondary oxidation processes. Its transformation varies from immature in the Lochkovian of the Lviv Basin to mature and overmature in the Emsian of the Lublin Basin.

2. In the Middle Devonian of the Lublin Basin, the TOC content varies from 0.00 to $0.62 \mathrm{wt} . \%$ (median $0.16 \mathrm{wt} . \%$ ) in the Eifelian, and from 0.03 to 1.63 wt.\% (median 0.18 wt. \%) in the Givetian. The organic matter is mature. In the Lviv Basin, the TOC content varies from 0.02 to 0.64 wt. $\%$ (median 0.08 wt. $\%$ ) in the Eifelian, and from 0.01 to 2.35 wt.\% (median 0.19 wt. \%) in the Givetian. The organic matter is immature in the Givetian, and mature in the Eifelian. Type-Il kerogen dominates.

3. In the Upper Devonian of the Lublin Basin, the TOC content varies from 0.02 to $2.44 \mathrm{wt} . \%$ (median $0.17 \mathrm{wt} . \%$ ) in the Frasnian, and from 0.00 to $2.62 \mathrm{wt} . \%$ (median $0.18 \mathrm{wt} \%$ ) in the Famennian. The organic matter is mature. In the Lviv Basin, the TOC content varies from 0.04 to 1.43 wt. $\%$ (median 0.08 wt. $\%$ ) in the Frasnian, and from 0.07 to 0.10 wt. \% (median 0.09 wt. \%) in the Famennian. The organic matter is mature in the Famennian, and overmature in the Frasnian. Type-II kerogen dominates.

4. Reservoir horizons in the Devonian of the Lublin and Lviv basins are developed in clastic, carbonate and sulphate rocks. Terrigenous reservoir rocks are composed of fine and medium-grained sandstones and fine- and coarse-grained siltstones. The pore space in the rocks is formed by intergranular spaces of 0.05 to $0.5 \mathrm{~mm}$ size in the Middle Devonian and from 0.08 to $1.3 \mathrm{~mm}$ in the Upper Devonian. Terrigenous rocks form gas-bearing horizons in the Middle Devonian (Eifelian and Givetian; Lopushany, Pelcha and Strutyn suites) in the Lokachi field of the Lviv Basin, and gas- and oil-bearing horizons in the Upper Devonian (Famennian; Hulcze Formation) in the Glinnik and Ciecierzyn fields of the Lublin Basin.

5. Carbonate rocks are represented by a wide range of lithological types from limestones to dolomites. Secondary processess formed fracture-like micro-cavities up to $0.5 \mathrm{~mm}$ in size and a high porosity. In carbonate reservoir rocks, fractures prevail, and pores are subordinate. Carbonate rocks form gas-bearing horizons in the Middle Devonian Komarów (Lublin Basin) and Lokachi (Lviv Basin) gas fields, as well as in the Frasnian in the Ciecierzyn and Mełgiew A and B fields (Lublin Basin).
6. Sulphate and carbonate-sulphate rocks are composed of anhydrites and dolomites with anhydrite concretions. They form a reservoir horizon in the Telatyń Formation in the Komarów field.

7. The natural hydrocarbon gas analysed, collected from the Middle and Upper Devonian reservoirs, vary in their molecular and isotopic compositions. Relatively ${ }^{13} \mathrm{C}$-depleted methane and ${ }^{13} \mathrm{C}$-enriched propane compared to ethane indicate that natural gas was not generated from a single source rock (multiple source) or has undergone post-generation alteration. Gas collected from the Upper Devonian of the Lublin Basin contains only insignificant amounts of microbial methane and was generated from Ordovician-Silurian Type-II kerogen. Gas from the Middle Devonian of the Lviv Basin includes a significant component of microbial methane and was generated from Middle and Upper Devonian mixed II/III kerogen of maturity from about 0.9 to $1.4 \%$ on the vitrinite reflectance scale.

8. Carbon dioxide occurs in the natural gas analysed in concentrations from 0.03 to $0.20 \mathrm{vol} \%$ and $\delta^{13} \mathrm{C}\left(\mathrm{CO}_{2}\right)$ values vary from -9.2 to $-8.1 \%$. The insignificant concentrations and stable carbon composition of carbon dioxide suggest that in the gas analysed from the Upper Devonian of the Lublin Basin (Ciecierzyn-1) a thermogenic component occurs, while in gas from the Middle Devonian of the Lviv Basin (Lokachi-27 and Lokachi-65) both thermogenic and microbial components can co-occur.

9. Molecular nitrogen occurs in the natural gas analysed in concentrations from 2.52 to $4.79 \%$ and $\delta^{15} \mathrm{~N}\left(\mathrm{~N}_{2}\right)$ values vary from -11.3 to $-7.0 \%$. The generally increasing trend of $\delta^{15} \mathrm{~N}\left(\mathrm{~N}_{2}\right)$ values with the growth of $\mathrm{N}_{2}$ concentration may suggest that molecular nitrogen was mainly generated during thermal transformation of organic matter and originated during destruction of $\mathrm{NH}_{4}$-rich illites of the clayey facies of Ordovician-Silurian strata in the Lublin (Ciecierzyn-1) and Lviv (Lokachi-27 and Lokachi-65) basins.

Acknowledgements. This geochemical study was undertaken as statutory research of the Faculty of Geology, Geophysics and Environmental Protection at the AGH University of Science and Technology in Kraków, project No. 11.11.140.626. The authors are grateful to Y. Koltun (Lviv, Ukraine), T. Peryt (Warsaw, Poland), M. Narkiewicz (Warsaw, Poland) and Anonymous Reviewer for many helpful comments on an earlier version of this manuscript. Analytical work by A. Kowalski, $\mathrm{T}$. Kowalski and $\mathrm{H}$. Zych from the AGH University of Science and Technology is gratefully acknowledged.

\section{REFERENCES}

Bernard, B.B., Brooks, J.M., Sackett, W.M., 1976. Natural gas seepage in the Gulf of Mexico. Earth and Planetary Science Letters, 31: 48-54.

Berner, U., Faber, E., 1996. Empirical carbon isotope/maturity relationships for gases from algal kerogens and terrigenous organic matter, based on dry, open-system pyrolysis. Organic Geochemistry, 24: 947-955.

Berner, U., Faber, E., 1997. Carbon isotope/maturity relationships for gases from algal kerogens and terrigenous organic matter Geologisches Jahrbuch, D 103: 129-145.

Chebanenko, I.I., Vishsnyakov, I.B., Vlasov, B.I., 1990 Geotektonika Volyno-Podolii (in Russian). Naukova Dumka, Kiev.
Chung, H.M., Gormly, J.R., Squires, R.M., 1988. Origin of gaseous hydrocarbons in subsurface environments: theoretical considerations of carbon isotope distribution. Chemical Geology, 71: 91-103.

Coleman, M. L., Shepherd, T.J., Durham, J.J., Rouse, J.E., Moore, G.R., 1982. Reduction of water with zinc for hydrogen isotope analysis. Analytical Chemistry, 54: 993-995.

Coplen, T.B., 2011. Guidelines and recommended terms for expression of stable-isotope-ratio and gas-ratio measurement results. Rapid Communications in Mass Spectrometry, 25: 2538-2560.

Dai, J., Song, Y., Dai, C., Wang, D., 1996. Geochemistry and accumulation of carbon dioxide gases in China. AAPG Bulletin, 80: $1615-1626$. 
Espitalié, J., Deroo, G., Marquis, F., 1985. La pyrolyse Rock-Eval et ses applications. Revue de l'Institut Français du Pétrole, 40-41: 563-579 and 755-784.

Fedyshyn, V.O. 1998. Atlas of Oil and Gas Fields of Ukraine. Ukrainian Oil and Gas Academy, Lviv.

Fischer, M., Botz, R., Schmidt, M., Rockenbauch, K., Garbe-Schönberg, D., Glodny, J., Gerling, P., Littke, R., 2006. Origins of $\mathrm{CO}_{2}$ in Permian carbonate reservoir rocks (Zechstein, Ca2) of the NW-German Basin (Lower Saxony). Chemical Geology, 227: 184-213.

Florkowski, T., 1985. Sample preparation for hydrogen isotope analysis by mass spectrometry. International Journal of Applied Radiation and Isotopes, 36: 991-992.

Galabuda, M., Krupskiy, Y., Pavlyuk, Î., Chepil, P., 2007. History of investigation of geological structure and oil and gas bearing perspectives of Volyno-Podillya (in Ukrainian with English abstract). Geologiya i Geokhimiya Goryuchykh Kopalyn, (3): 5-18.

Garbacik, Z., 1989. Dokumentacja wynikowa odwiertu poszukiwawczego: Glinnik-2 (in Polish). NAG PIG, Warszawa (Inw. 131902).

Gerling, P., Idiz, E., Everlien, G., Sohns, E., 1997. New aspects on the origin of nitrogen in natural gas in Northern Germany. Geologisches Jahrbuch, D103: 65-84.

Gutsalo, L.K., Plotnikov, A.M., 1981 Izotopnyi sostav ugleroda v sistemie $\mathrm{CH}_{4}-\mathrm{CO}_{2}$ kak kriteriy proiskhozhdeniya metana i dvuokisi ugleroda $v$ prirodnykh gazakh Zemli (in Russian). Doklady Akademyi Nauk SSSR, 259: 470-473.

Helcel-Weil, M., Dzięgielowski, J., 2003. Lublin Basin - petroleum prospecting results and their importance for future exploration. Przegląd Geologiczny, 51: 764-770.

Helcel-Weil, M., Dzięgielowski, J., Florek, R., Maksym, A., Słyś, M., 2007. The Lublin Basin: petroleum exploration results and their importance for future prospects (in Polish with English summary). Biuletyn Państwowego Instytutu Geologicznego, 411: $51-62$

Hinrichs, K-U., Hayes, J.M., Bach, W., Spivack, A.J., Hmelo, L.R., Holm, N.G., Johnson, C.G., Sylva, S.P., 2006. Biological formation of ethane and propane in the deep marine sediments. Proceedings of the National Academy of Sciences, 103: 14684-14689.

Hutcheon, I., Abercrombie, H., 1990. Carbon dioxide in clastic rocks and silicate hydrolysis. Geology, 18: 541-544.

Jenden, P.D., Hilton, D.R., Kaplan, I.R., Craig, H., 1993. Abiogenic hydrocarbons and mantle helium in oil and gas fields. U.S. Geological Survey Professional Papers, 1570: 31-56.

Karnkowski, P., 1999. Oil and Gas Deposits in Poland. Ed. Geological Society "Geos", Kraków.

Khizhniakov, A.V., 1975. O perspektivakh poiskov nefti i gaza vo Lvovskom progibe (in Russian). In: Zakonomernosti obrazovaniya i razmieshcheniya promyshlennykh mestorozhdeniy nefti i gaza. Naukova Dumka, Kiev.

Kosakowski, P., Więcław, D., Kotarba, M.J., Kowalski, A., 2012a. Hydrocarbon potential of the Mesozoic strata between Kraków and Rzeszów (SE Poland). Geological Quarterly, 56 (1): 139-152.

Kosakowski, P., Więcław, D., Kowalski, A., Koltun, Y.V., 2012b. Hydrocarbon potential of the Jurassic and Cretaceous source rocks between Tarnogród and Stryi (SE Poland and western Ukraine). Geologica Carpathica, 63: 319-333.

Kotarba, M., 1988. Geochemical criteria for origin of natural gases accumulated in the Upper Carboniferous coal-seam-bearing formations in Wałbrzych Coal Basin (in Polish with English summary). Zesz. Nauk. Akad. Górn.-Hutn. (Academy Mining and Metallurgy Bulletin), Geologia (Geology), 42: 1-119.

Kotarba, M.J., 2012. Origin of natural gases in the Paleozoic-Mesozoic basement of the Polish Carpathian Foredeep. Geologica Carpathica, 63: 307-318.

Kotarba, M.J., Lewan, M.D., 2004. Characterizing thermogenic coalbed gas from Polish coals of different ranks by hydrous pyrolysis. Organic Geochemistry, 35: 615-646.
Kotarba, M.J., Lewan, M.D., 2013. Sources of natural gases in Middle Cambrian reservoirs in Polish and Lithuanian Baltic Basin as determined by stable isotopes and hydrous pyrolysis of Lower Palaeozoic source rocks. Chemical Geology, 345: 62-76.

Kotarba, M.J., Rice, D.D., 2001. Composition and origin of coalbed gases in the Lower Silesian Basin, northwestern Poland. Applied Geochemistry, 16: 895-910.

Kotarba, M.J., Kosakowski, P., Kowalski, A., Więcław, D., 1998 Preliminary geochemical characteristics of organic matter and hydrocarbon potential of the Devonian deposits in the Radom-Lublin and Pomerania areas (in Polish with English summary) Prace Państwowego Instytutu Geologicznego, 165: 207-214.

Kotarba, M.J., Więcław, D., Kosakowski, P., Zacharski, J., Kowalski, A., 2003. Evaluation of source rock and petroleum potential of Middle Jurassic strata in the south-eastern part of Poland (in Polish with English abstract). Przegląd Geologiczny, 51: 1031-1040.

Kotarba, M.J., Więcław, D., Kosakowski, P., Kowalski, A., Kowalski, T., 2005. Skały macierzyste (in Polish). In: Lokalizacja geologiczna i system naftowy w basenie lubelskim oraz perspektywy poszukiwawcze (ed. M. Narkiewicz). Unpublished report, Archive of Polish Geological Institute - National Research Institute, Warsaw, Chapter 6A: $48 \mathrm{p}$.

Kotarba, M.J., Curtis, J.B., Lewan, M.D., 2009. Comparison of natural gases accumulated in Oligocene strata with hydrous pyrolysis gases from Menilite Shales of the Polish Outer Carpathians. Organic Geochemistry, 40: 769-783.

Kotarba, M.J., Więcław, D., Kosakowski, P., Wróbel, M., Buła, Z., Matyszkiewicz, J., Krajewski, M., Kowalski, A., Koltun, Y.V., 2011. Petroleum systems and prospectives of hydrocarbon exploration in the Palaeozoic-Mesozoic basement (SE Poland and western Ukraine). Annales Societatis Geologorum Poloniae, 81: 487-522.

Krooss, B.M., Littke, R., Müller, B., Frielingsdorf, J., Schwochau, K., Idiz, E.F., 1995. Generation of nitrogen and methane from sedimentary organic matter: implications on the dynamics of natural gas accumulations. Chemical Geology, 126: 291-318.

Krooss, B.M., Friberg, L., Gensterblum, Y., Hollenstein, J., Prinz, D., Littke, R., 2005. Investigation of the pyrolytic liberation of molecular nitrogen from Paleozoic sedimentary rocks. International Journal of Earth Sciences, 94: 1023-1038.

Kruglov, C.C., Tsypko, A.K., 1988. Tektonika Ukrainy (in Russian). Nedra, Moscow.

Krupskiy, J.Z., 2001. Geodynamic conditions of forming and oil and gas bearing of Carpathian and Volyno-Podillya regions of Ukraine (in Ukrainian with English abstract). UkrDGRI, Kiev.

Krupskiy, J.Z., Kurovets, I.M., Senkovskiy, J.M., Michailov, W.A., Chepil, P.M., Drygant, D.M., Shlapinskiy, W.E., Koltun, J.W., Chepil, W.P., Kurovets, S.S., Bodlak, W.P., 2014. Netradytsiyni dzherela vuglevodniv v Ukrayiny (in Ukrainian) Book II: Western gas-bearing region. Nika-Centre, Kiev.

Krzywiec, P., Mazur, S., Gągała, Ł, Kufrasa, M., Lewandowski, M., Malinowski, M., Buffenmyer, V., 2017. Late Carboniferous thin-skinned compressional deformation above the SW edge of the East European Craton as revealed by reflection seismic and potential fields data - correlations with the Variscides and the Appalachians. GSA Memoir, 213: 1-20, doi:10.1130/2017.1213(14)

Lillis, P.G., 2007. Upper Cretaceous microbial petroleum systems in north-central Montana. The Mountain Geologist, 44: 11-35.

Littke, R., Krooss, B.M., Idiz, E.F, Frielingsdorf, J., 1995. Molecular nitrogen in natural gas accumulations: generation from sedimentary organic matter at high temperatures. AAPG Bulletin, 79: 410-430.

Lüders, V., Reutel, Ch., Hoth, P., Banks, D.A., Mingram, B., Pettke, T., 2005. Fluid and gas migration in the North German Basin: fluid inclusion and stable isotope constrains. International Journal of Earth Sciences, 94: 990-1009. 
Medvedyev, A.P., 1979. Priroda doalpiyskoy struktury Volyno-Podolii i smezhnykh rayonov (in Russian). Naukova Dumka, Kiev.

Miłaczewski, L., 1981. The Devonian of the south-eastern part of the Radom-Lublin area (in Polish with English summary). Prace Instytutu Geologicznego, 51: 1-90.

Miłaczewski, L., 2010. Lithofacies-paleothickness maps of Middle Devonian, Frasnian and Famennian, 1:200,000. In Paleogeological Atlas of the sub-Permian Paleozoic of the East-European Craton in Poland and Neighboring Areas (ed Z. Modliński). Państwowy Instytut Geologiczny - Państwowy Instytut Badawczy, Warszawa.

Milkov, A.V., 2011. Worldwide distribution and significance of secondary microbial methane formed during petroleum biodegradation in conventional reservoirs. Organic Geochemistry, 42: 184-207.

Mingram, B., Hoth, P., Lüders, V., Harlov, D., 2005. The significance of fixed ammonium in Palaeozoic sediments for the generation of nitrogen-rich natural gases in the North German Basin. International Journal of Earth Sciences, 94: 1010-1022.

Modzelewski, R., 1999. Dokumentacja geologiczna złoża gazu ziemnego Ciecierzyn (in Polish). NAG PIG, Warszawa (Inw. $2501 / 2000$ )

Narkiewicz, M., 2007. Development and inversion of Devonian and Carboniferous basins in the eastern part of the Variscan foreland (Poland). Geological Quarterly, 51 (3): 231-256.

Narkiewicz, M., 2011. Lithostratigraphy, depositional systems and transgressive-regressive cycles in the Devonian of the Lublin Basin (south-eastern Poland) (in Polish with English summary). Prace Państwowego Instytutu Geologicznego, 196: 53-146.

Narkiewicz, M., Narkiewicz, K., Turnau, E., 2011. Devonian depositional development of the Łysogóry-Radom and Lublin Basins (South-eastern Poland) (in Polish with English summary). Prace Państwowego Instytutu Geologicznego, 196: 289-318.

Narkiewicz, M., Maksym, A., Malinowski, M., Grad, M., Guterch, A., Petecki, Z., Probulski, J., Janik, T., Majdański, M., Środa P., Czuba, W., Gaczyński, E., Jankowski, L., 2015 Transcurrent nature of the Teisseyre-Tornquist Zone in Centra Europe: results of the POLCRUST-01 deep reflection seismic profile. International Journal of Earth Sciences, 104: 775-796.

Peters, K.E, Cassa, M.R., 1994. Applied source rock geochemistry. AAPG Memoir, 60: 93-120.

Pomyanovskaya, G.M., 1974. The Volyn-Podolian outskirt of the East-European Platform (in Russian with English summary). In Stratigraphy of the Ukrainian SSR, 4, Part 2, Devonian: 36-120. Naukova Dumka, Kiev.

Pożaryski, W., Dembowski, Z., 1983. Mapa geologiczna Polski krajów ościennych bez utworów kenozoicznych, mezozoicznych i permskich, 1:1 000000 (in Polish). Instytut Geologiczny, Warszawa.

Radkovets, N., 2015. The Silurian of southwestern margin of the East European Platform (Ukraine, Moldova and Romania): lithofacies and palaeoenvironments. Geological Quarterly, 59 (1): 105-118

Radkovets, N., 2016. Lower Devonian lithofacies and palaeoenvironments in the southwestern margin of the East Eu- ropean Platform (Ukraine, Moldova and Romania). Estonian Journal of Earth Sciences, 65: 207-220.

Rooney, M.A., Claypool, G.E., Chung, H.M., 1995. Modeling thermogenic gas generation using carbon isotope ratios of natural gas hydrocarbons. Chemical Geology, 126: 219-232.

Rzeźnik, M., 2012. Dodatek nr 1 do dokumentacji geologicznej złoża ropy naftowej "Glinnik" w kat. B w miejsc. Amelin, Meszno (in Polish). NAG PIG, Warszawa (Inw. 972/2013).

Schoell, M., 1988. Multiple origins of methane in the Earth. Chemical Geology, 71: 1-10.

Smith, J.T., Ehrenberg, S.N., 1989. Correlation of carbon dioxide abundance with temperature in clastic hydrocarbon reservoirs: relationship to inorganic chemical equilibrium. Marine and $\mathrm{Pe}$ troleum Geology, 6: 129-135.

Stachurski, Z., Karnkowski, K., Kruczak, E., 1985. Dokumentacja wynikowa otworu badawczego: Ciecierzyn-1. NAG PIG, Warszawa (Inw. 129693).

Stahl, W., 1977. Carbon and nitrogen isotopes in hydrocarbon research and exploration. Chemical Geology, 20: 121-149.

Vashchenko, V.O., Turchynova, S.M., Turchynov, I.I., Poliha, G.G., 2007. Derzhavna geologichna karta Ukrainy mashtabu 1:200 000. Karpatska seria. Arkush M-35-XXV (Ivano-Frankivsk) (in Ukrainian). UkrDGRI, Kiev.

Whiticar, M.J., 1994. Correlation of natural gases with their sources. AAPG Memoir, 60: 261-283.

Whiticar, M.J., Faber, E., Schoell, M., 1986. Biogenic methane formation in marine and freshwater environments: $\mathrm{CO}_{2}$ reduction vs. acetate fermentation-Isotope evidence. Geochimica et Cosmochimica Acta, 50: 693-709.

Więcław, D., Kotarba, M.J., Kowalski, A., Kosakowski, P., 2011 Habitat and hydrocarbon potential of the Palaeozoic source rocks in the Kraków-Rzeszów area (SE Poland). Annales Societatis Geologorum Poloniae, 81: 375-394.

Więcław, D., Kosakowski, P., Kotarba, M.J., Kowalski, A., Koltun, Y.V., 2012. Assessment of hydrocarbon potential of the Lower Palaeozoic strata in the Tarnogród-Stryi area (SE Poland and western Ukraine). Annales Societatis Geologorum Poloniae, 82: 65-80.

Zhang, T., Zhang, M., Bai, B., Wang, X., Li, L., 2008. Origin and accumulation of carbon dioxide in the Huanghua depression, Bohai Bay Basin, China. AAPG Bulletin, 92: 341-358.

Zhu, Y., Shi, B., Fang, C., 2000. The isotopic compositions of molecular nitrogen: implications on their origins in natural gas accumulations. Chemical Geology, 164: 321-330.

Zou, Y.-R., Cai, Y., Zhang, Ch., Zhang, X., Peng, P., 2007. Variations of natural gas carbon isotope-type curves and their interpretation - A case study. Organic Geochemistry, 38: 1398-1415

Zelichowski, A., 1972. Evolution of the geological structure of the area between the Góry Świętokrzyskie and the river Bug (in Polish with English summary). Biuletyn Instytutu Geologicznego, 263: 1-97

Żelichowski, A., Kozłowski, S., 1983. Atlas of the Geological Structure and Mineral Deposits in the Lublin Region. Wyd. Geol., Warszawa. 